This is the author's copy of the publication as archived with the DLR's electronic library at http://elib.dlr.de . Please consult the original publication for citation, see e.g. https://ieeexplore.ieee.org/abstract/document/8952557

\title{
Validation and Update of an Aeroservoelastic Model based on Flight Test Data
}

\author{
Matthias Wüstenhagen, Özge Süelözgen, Lukas Ackermann, Julius Bartasevicius
}

An unmanned aircraft serves as a testing platform to demonstrate the benefits of active flutter suppression. The mathematical model representing the structural dynamics and aerodynamics of the demonstrator aircraft is described. Based on flight test data the rigid body model is updated in two steps. At first the aircraft states and sensor measurements are reconstructed. Subsequently, the output error method is used to estimate the desired aerodynamic parameters. The mathematical model with updated parameters appropriately describes the longitudinal and lateral aircraft dynamics. Furthermore, a comparison of the aeroelastic mode shapes of the derived model shows good agreement, which is even improved when updating aerodynamic damping values obtained from flight tests.

\section{Copyright Notice}

(C2021 IEEE. Personal use of this material is permitted. Permission from IEEE must be obtained for all other uses, in any current or future media, including reprinting/republishing this material for advertising or promotional purposes,creating new collective works, for resale or redistribution to servers or lists, or reuse of any copyrighted component of this work in other works.

Wüstenhagen, Matthias; Süelözgen, Özge; Ackermann, Lukas; Bartaševičius, Julius, "Validation and Update of an Aeroservoelastic Model based on Flight Test Data“, 2021 IEEE Aerospace Conference (50100), Big Sky, MT, USA, 2021, doi:

10.1109/AERO50100.2021.9438354. 


\section{Validation and Update of an Aeroservoelastic Model based on Flight Test Data}

\author{
Matthias Wüstenhagen \\ German Aerospace Center \\ Institute of System Dynamics and Control \\ Münchener Strasse 20 \\ 82234 Wessling, Germany \\ $+49815328-2867$ \\ matthias.wuestenhagen@dlr.de \\ Lukas Ackermann \\ German Aerospace Center \\ Institute of System Dynamics and Control \\ Münchener Strasse 20 \\ 82234 Wessling, Germany \\ +498153 28-2867 \\ lukas.ackermann@dlr.de
}

\author{
Özge Süelözgen \\ German Aerospace Center \\ Institute of System Dynamics and Control \\ Münchener Strasse 20 \\ 82234 Wessling, Germany \\ +498153 28-2904 \\ oezge.sueeloezgen@dlr.de \\ Julius Bartaševičius \\ Technical University of Munich \\ Institute of Aircraft Design \\ Boltzmannstrasse 15 \\ 85748 Garching b. Munich \\ $+4989289-15797$ \\ julius.bartasevicius@tum.de
}

\begin{abstract}
An unmanned aircraft serves as a testing platform to demonstrate the benefits of active flutter suppression. The mathematical model representing the structural dynamics and aerodynamics of the demonstrator aircraft is described. Based on flight test data the rigid body model is updated in two steps. At first the aircraft states and sensor measurements are reconstructed. Subsequently, the output error method is used to estimate the desired aerodynamic parameters. The mathematical model with updated parameters appropriately describes the longitudinal and lateral aircraft dynamics. Furthermore, a comparison of the aeroelastic mode shapes of the derived model shows good agreement, which is even improved when updating aerodynamic damping values obtained from flight tests.
\end{abstract}

\section{TABle OF Contents}

1. INTRODUCTION..............................1

2. Aeroservoelastic Aircraft Modelling .....2

3. Flight TESTING ........................

4. UPDATE OF THE RIGID BODY MODEL ...........8

5. FLEXIBLE MOdE IDENTIFICATION ............ 11

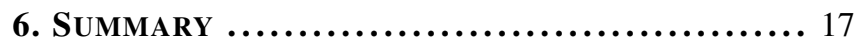

ACKNOWLEDGMENTS ...................... 17

REFERENCES ............................. 17

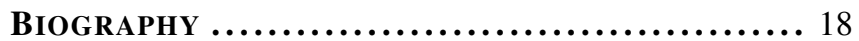

\section{INTRODUCTION}

The fuel costs account for the greatest portion of the operating costs of today's aircraft. Therefore, fuel efficiency is one of the most important aspect of new aircraft design concepts [1]. Lightweight wing structures and higher wing aspect ratios hold great potential to increase the fuel efficiency and make flying more economic. These modifications, however, lead to a higher wing flexibility and the flutter speed decreases. Flutter is a vibrational instability of the wings, which can result in catastrophic structural failure. It generally involves the coupling between wing bending and torsion [2,3]. Active flutter suppression offers the opportunity to prevent flutter and guarantee stability within the entire flight envelope. As part of

978-1-7281-7436-5/21/\$31.00 @2021 IEEE the Horizon 2020 projects Flutter Free FLight Envelope eXpansion for ecOnomic Performance improvement (FLEXOP) [4] and FLight Phase Adaptive Aero-Servo-Elastic aircraft Design methods (FLiPASED) [5] active flutter suppression methods are developed and tested on the demonstrator aircraft shown in Figure 1. A flutter controller, which controls the

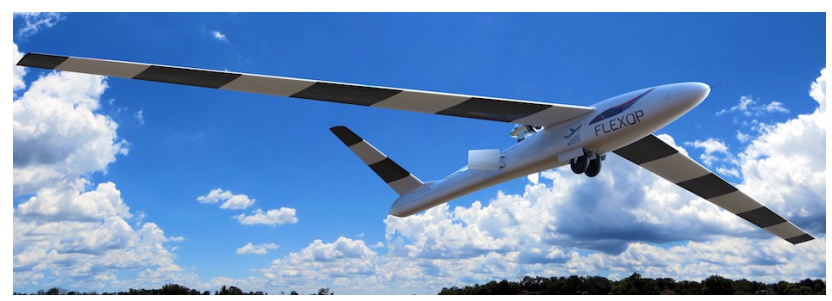

Figure 1: FLiPASED demonstrator aircraft [4]

actuators of the ailerons, forms the core of the flutter suppression system. It is developed based on aeroelastic models. It is therefore essential to derive sophisticated aeroelastic models beforehand. More insight into the design of an active flutter controller is given in Ref. 6-8.

Before the aircraft is tested beyond its open-loop flutter speed, it is tested with a rather stiff set of wings featuring a much higher open-loop flutter speed. This offers the opportunity to undergo the entire process of modelling and flight testing without the risk of harming the aircraft. The presented work solely presents results based on the "stiffwing-configuration". Applying the process to the fluttercritical configuration is part of research yet to be conducted.

The aeroelastic modelling process within the FLEXOP project is described in Figure 2. A finite element (FE) model represents the structural dynamics of the demonstrator. As the FE model features many degrees of freedom (DOF), it is unsuitable for flutter control design. The DOF are decreased by means of the Guyan reduction. However, the relevant dynamics are preserved. A condensed model with significantly less DOF is the result. Based on this model the equations of motion (EOM) describing the rigid and flexible aircraft motion are formulated [9].

The aerodynamic model is built of trapezoidal aerodynamic boxes, which represent the lifting surfaces. Vortices or 


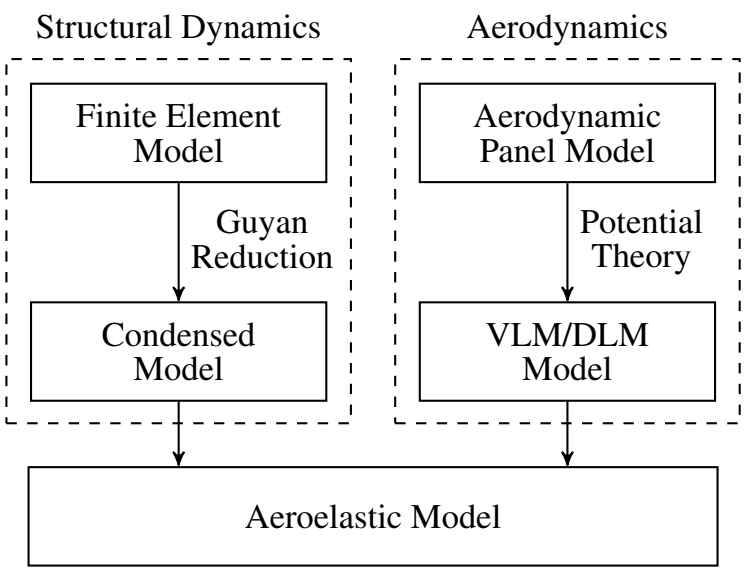

Figure 2: Aeroelastic modeling process of the demonstrator aircraft

doublets from potential theroy are applied to the boxes approximating the demonstrator flow field. The application with vortices is called vortex lattice method (VLM), while with doublets it is known as the doublet lattice method (DLM), respectively. The VLM represents steady aerodynamics. To extend the aerodynamic model for unsteady aerodynamic effects the DLM is applied $[2,10]$.

The interconnection between the structural dynamics and aerodynamics is carried out through splining, which results in an aeroelastic model. The entire aircraft modelling process is described in greater depth in Section 2.

For the analysis and adaption of the proposed aeroelastic model several flight test manoeuvres are defined. In this context, the rigid body and flexible body motion of the aircraft are treated separately. With respect to the rigid body model the two-step method (TSM) is applied. The first step is the flight path reconstruction (FPR). At this point it is assumed, that the most reliable measurements are the translational accelerations and angular rates. These serve as inputs to the flight mechanical EOM. Aircraft states and sensor readings that are prone to disturbances can be determined. The reconstructed signals help to make the parameter estimation more reliable. The desired parameters are the aerodynamic parameters accounting for the rigid body motion of the aircraft. These are updated in an optimization using the output error method (OEM) [11].

Inertial measurement units (IMUs) are attached to the aircraft wings, in order to record the deformation of the wings. The analysis of the IMU data provides information on the aeroelastic mode shapes that the wings are exposed to in flight. Subsequently, the aeroelastic model is updated.

\section{Aeroservoelastic Aircraft MODELLING}

The overall aeroservoelastic model is built of several submodels, as depicted in Figure 3. The core forms the aeroelastic system coupling the aerodynamics and structural dynamics. Adding control related systems, like actuators, sensors and the controller itself, completes the aeroservoelastic model. In the following, the subject of investigation is the update of the aeroelastic model, therefore the structural dynamics and the aerodynamics are described further.

\section{Structural Dynamics}

The structural dynamics of the demonstrator aircraft are divided by their contributions to the rigid and flexible body motion. The rigid body dynamics basically describe the manoeuvre characteristics of the aircraft. The flexible body dynamics represent the aircraft motion due to its flexible structure. While the rigid body dynamics are described in nonlinear form, the equation of the flexible body dynamics is defined linearly. A detailed FE model serves as basis for the structural model of the aircraft. The process of generating the FE model and its condensed version is described below. Subsequently, the EOM are applied to the condensed model.

Finite Element Model-The aircraft structural FE model comprises the wing, fuselage and empennage. It is shown in Figure 4. The wings are built of beam, surface and solid elements. Rigid body interpolation elements are added at predefined locations throughout the wing to facilitate the required model reduction. The fuselage consists of beam elements only. The equivalent beam stiffnesses are obtained utilizing the cross sections of the fuselage hull at different sections and the lay-up of the hull [13]. The mass is then lumped at the two beam nodes. The V-tail empennage FE model is shell-element-based [9].

Given that the FE model of the wing is of very high fidelity (more than 600000 nodes), a Guyan reduction, also called condensation, is performed reducing the mass and stiffness matrices to less than 200 nodes in the condensed model $[9,14]$.

Equations of Motion-The structural dynamics are described by the EOM based on an equilibrium of forces and moments. They describe the behaviour of the aircraft due to external loads $P_{g}^{\text {ext }}$, defined by

$$
P_{g}^{\text {ext }}=P_{g}^{\text {eng }}+P_{g}^{\text {aero }}
$$

It originates from the thrust $P_{g}^{\text {eng }}$ and aerodynamic loads $P_{g}^{\text {aero }}$. For simplification, the following assumptions are made [9]:

1. As the earth rotation can be neglected, the inertial reference system is earth fixed [10].

2. Gravity is constant over the airframe [15].

3. The deformations of the airframe are considered to be small which allows the use of linear elastic theory defined by Hooke's law [10].

4. Due to small deformations of the aircraft structure, the aircraft mass moment of inertia $J_{b}$ remains unchanged [10].

5. As the structural deformations are small, loads act on the undeformed airframe [15].

6. The eigenvectors of the modal analysis are orthogonal, because of which the total structural deformation can be written as a linear combination of the modal deflections [15]. 7. The rigid body and flexible body EOM are considered to be decoupled [15].

Rigid Body Dynamics - For the derivation of the nonlinear flight mechanical EOM, the aircraft is considered a rigid body with constant mass $m_{b}$ and constant mass moment of inertia $J_{b}$. Thus, the aircraft rigid body motion is given by the Newton-Euler EOM [16]

$$
\left[\begin{array}{c}
m_{b}\left(\dot{V}_{b}+\Omega_{b} \times V_{b}-T_{b e} g_{e}\right) \\
J_{b} \dot{\Omega}_{b}+\Omega_{b} \times\left(J_{b} \Omega_{b}\right)
\end{array}\right]=\underbrace{\Phi_{g b}^{T} P_{g}^{\text {ext }}}_{P_{b}^{\text {ext }}}=\left[\begin{array}{c}
F \\
M
\end{array}\right] .
$$

The translational and angular velocities of the aircraft with respect to the body frame of reference are defined by $V_{b}$ and 


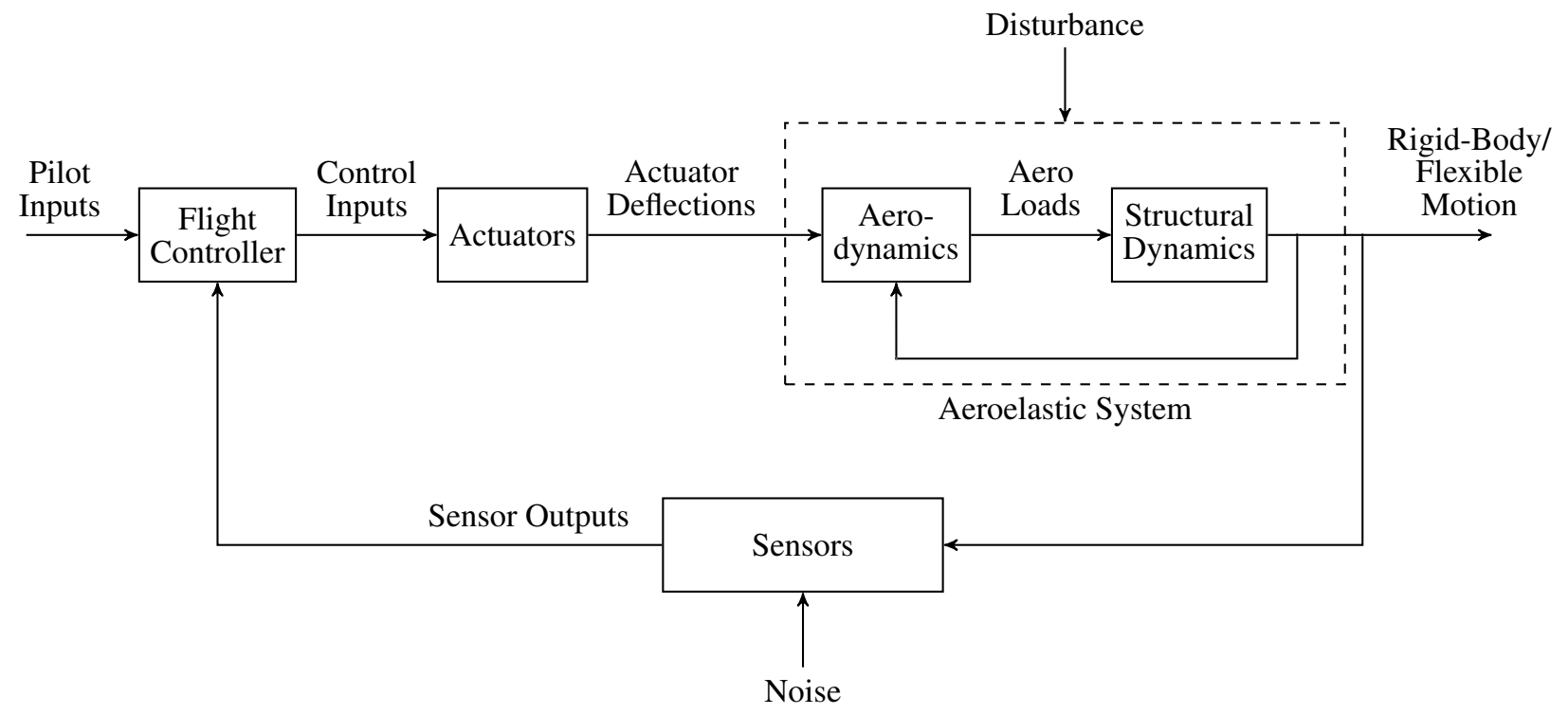

Figure 3: Aeroservoelastic system $[9,12]$

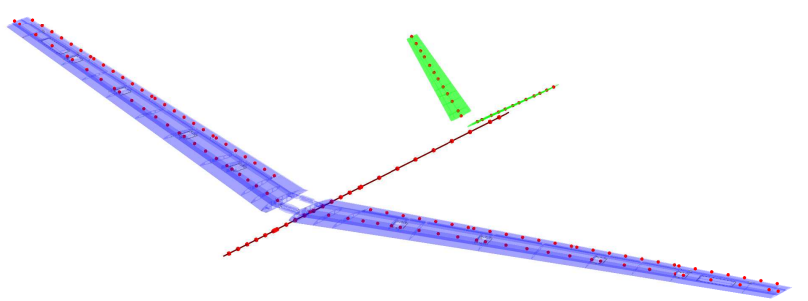

Figure 4: Full FE model of the FLEXOP demonstrator aircraft [9]

$\Omega_{b}$. The vector $g_{e}$ represents the gravitational acceleration, which is transformed with $T_{b e}$ from the earth-fixed to the body-fixed frame of reference. Multiplication of the loads $P_{g}^{\text {ext }}$ with matrix $\Phi_{g b}^{T}$ transforms them into the rigid body frame $[9,10]$.

Flexible Body Dynamics - The displacements due to the aircraft flexibility are assumed to be small. Therefore, linear elastic theory is applied to define the flexible body motion. The correlation between external loads $P_{g}^{\text {ext }}$ and generalized coordinates $u_{f}$ representing the modal deformation of the structure is given by

$$
M_{f f} \ddot{u}_{f}+B_{f f} \dot{u}_{f}+K_{f f} u_{f}=\underbrace{\Phi_{g f}^{T} P_{g}^{\mathrm{ext}}(t)}_{P_{f}^{\text {ext }}(t)} .
$$

The matrices $M_{f f}, B_{f f}$ and $K_{f f}$ are the modal masses, dampings and stiffnesses. The modal matrix $\Phi_{g f}$ contains the eigenvectors of the structural modes sorted by frequency [10]. Typically, higher frequency modes contribute less to the flexible body motion. Consequently, modal truncation is applied to reduce the DOF significantly by considering only the most relevant eigenmodes [9].

\section{Aerodynamics}

The aerodynamic loads are the major external loads acting on the aircraft structure. Their calculation is either based on the VLM representing steady aerodynamics or the DLM introducing additionally unsteady aerodynamic effects. Both methods are based on a panel model.

Panel Model-The lifting surfaces are discretised by several trapezoidal-shaped panels, so-called aerodynamic boxes shown in Figure 5. Of note is the panel model for the

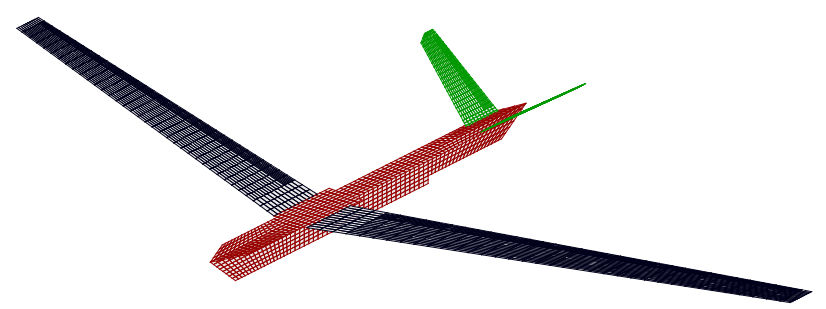

Figure 5: Aerodynamic boxes of the FLEXOP demonstrator aircraft [9]

fuselage. The wetted areas of the fuselage are projected onto a T-cruciform shaped panel model. Although this is a vast simplification, the fuselage aerodynamics are modelled quite accurately with respect to higher-order CFD simulations.

Steady Aerodynamics - The VLM is used for steady aerodynamics. As depicted in Figure 6a, each aerodynamic box of the panel model possesses a horseshoe vortex at point $l$ on the quarter-chord line. As the Helmholtz theorem states, the vortex is shed downstream to infinity at the side edges of the box. For each aerodynamic box the Pistolesi Theorem needs to be met, stating that there is no perpendicular flow through the control point $j$ at the three-quarter-chord line. Therefore, the induced velocity at the control point needs to equalize the perpendicular component of the incoming flow, like shown in Figure 6b. Using the Biot-Savart law the induced velocities $v_{j}$ caused by the circulation strengths $\Gamma_{j}$ of the horseshoe vortices are determined by

$$
v_{j}=A_{j j} \Gamma_{j}
$$




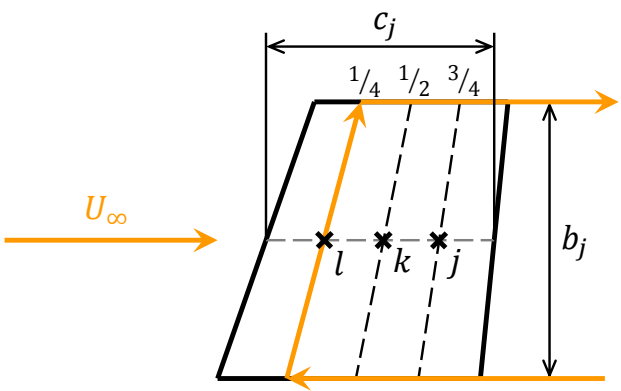

(a) Top view

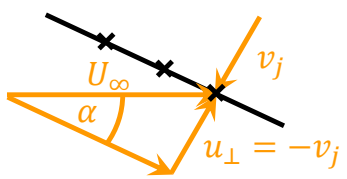

(b) Side view

Figure 6: Schematic drawing of an aerodynamic box [9]

The matrix $A_{j j}$ contains the contribution of all vortices to the induced velocities of each individual aerodynamic box. Inverting $A_{j j}$ and multiplying with $2 / c_{j}$, where $c_{j}$ is the chord length of the respective aerodynamic box, yields the aerodynamic influence coefficient (AIC) matrix $Q_{j j}$. If the aerodynamics are steady, it is considered constant. The pressure coefficients $\Delta c_{p j}$ of the panels are then determined by

$$
\Delta c_{p j}=Q_{j j} w_{j}
$$

where the downwash $w_{j}=\frac{v_{j}}{U_{\infty}}$ is the velocity $v_{j}$ normalized with the flight speed $U_{\infty}$. For small angles of attack $\alpha_{j}$ at a panel, $w_{j}$ is assumed to be equal $\alpha_{j}$, i.e. $w_{j}=\sin \left(\alpha_{j}\right) \approx \alpha_{j}$.

The downwash $w_{j}$ comprises different aerodynamic contributions. It is affected by a rigid body motion of the aircraft with

$$
w_{j b_{1}}=\frac{c_{r}}{2 U_{\infty}} D_{j k, 2} \Phi_{k a} T_{a b}\left[\begin{array}{c}
V_{b} \\
\Omega_{b}
\end{array}\right]
$$

The vector $\left[V_{b}^{T} \Omega_{b}^{T}\right]^{T}$ contains the rigid body velocities $V_{b}$ and angular rates $\Omega_{b}$ and is transformed to the aerodynamic centre by means of $T_{a b}$. Subsequently, the respective motion of each panel reference point $k$ is calculated by multiplying $\Phi_{k a}$. The resulting contribution to the downwash is then determined by multiplication with the matrix $D_{j k, 2}$ and factorisation with $\frac{c_{r}}{2 U_{\infty}}$, where $c_{r}$ depicts the reference chord length. Further details on the determination of the contributions of the downwash can be found in Ref. 10. Under the assumption of small angles Equation (6) can be rearranged to

$$
\begin{aligned}
& w_{j b_{1}}=\frac{c_{r}}{2} D_{j k, 2} \Phi_{k a} {\left[\begin{array}{c}
\cos \alpha_{a} \cos \beta_{a} \\
\sin \beta_{a} \\
\cos \beta_{a} \sin \alpha_{a} \\
p_{a} / U_{\infty} \\
q_{a} / U_{\infty} \\
r_{a} / U_{\infty}
\end{array}\right] } \\
& \approx \frac{c_{r}}{2} D_{j k, 2} \Phi_{k a}\left[\begin{array}{c}
1 \\
\beta_{a} \\
\alpha_{a} \\
p_{a} / U_{\infty} \\
q_{a} / U_{\infty} \\
r_{a} / U_{\infty}
\end{array}\right] .
\end{aligned}
$$

It can be seen that, besides the angular rates $p_{a}, q_{a}$ and $r_{a}$, the downwash is affected by the sideslip angle $\beta_{a}$ and the angle of attack $\alpha_{a}$. The "1" in the vector represents a constant contribution to the downwash. This gives the opportunity to add the downwash caused by effects like camber and twist by adaptation of the first column of $\Phi_{k a}$. As a first step, it is updated based on a steady computational fluid dynamics (CFD) calculation.

The deflection of the control surfaces $u_{x}$ is taken into account by changes in the downwash

$$
w_{j x_{0}}=D_{j k, 1} \Phi_{k x} u_{x} .
$$

The matrix $\Phi_{k x}$ links control surface deflections to the corresponding aerodynamic boxes. The differentiation matrix $D_{j k, 1}$ then relates a displacement of the panel reference point $k$ to the downwash $w_{j}$. Besides, the control surface deflection rate alters the lift, which can be accounted for by

$$
w_{j x_{1}}=\frac{c_{r}}{2 U_{\infty}} D_{j k, 2} \Phi_{k x} \dot{u}_{x}
$$

Introducing aeroelastic effects extends the model to a flexible aircraft. Comparable to the control surfaces, the flexible deflection $u_{f}$ and deflection rate $\dot{u}_{f}$ of the aircraft contribute with

$$
\begin{aligned}
& w_{j f_{0}}=D_{j k, 1} T_{k g} \Phi_{g f} u_{f} \\
& w_{j f_{1}}=\frac{c_{r}}{2 U_{\infty}} D_{j k, 2} T_{k g} \Phi_{g f} \dot{u}_{f}
\end{aligned}
$$

to the downwash, where the matrix $T_{k g}$ transforms structural grid deflections and deflection rates to the aerodynamic boxes [10].

Finally, the total downwash is the sum of the specified contributions given by [9]

$$
w_{j}=w_{j b_{1}}+w_{j x_{0}}+w_{j x_{1}}+w_{j f_{0}}+w_{j f_{1}} .
$$

The aerodynamic model does not include pressure contributions due to structural thickness. These are not associated with the downwash but with the tangential flow of a panel, which is not part of the proposed approach. As a consequence forces in the aircraft $\mathrm{x}$-direction are neglected, which is why the roll-yaw coupling of the aircraft for example is not accurately represented [17]. It is investigated, on how to extend the proposed model for forces in x-direction. Nevertheless, as a first step a drag model of the rigid body model was added. The drag coefficient $c_{D}$ is calculated by means of a preliminary drag polar providing a force in $\mathrm{x}$-direction [18]. 
Unsteady Aerodynamics-When applying the DLM the aerodynamics are widened to account for unsteady aerodynamic effects. An airfoil, that is suddenly moved forward at an angle of attack, creates circulation. Since the Helmholtz theorem states that the total circulation has to stay constant, a vortex of the same strength but switched sign is shed from the trailing edge. As it moves downstream it loses its influence on the airfoil. Therefore, with increasing time the flow converges to the steady condition. This lag is caused by unsteady aerodynamics [19].

To apply unsteady aerodynamics to the panel model, doublets instead of horseshoe vortices are placed at the quarter-chord line of each aerodynamic box. The pressure coefficient is then determined by

$$
\Delta c_{p j}(k)=Q_{j j}(k) w_{j}(k)
$$

in the reduced frequency domain, where the dimensionless reduced frequency $k$ is

$$
k=\omega \frac{c_{r}}{2 U_{\infty}}
$$

and $\omega$ is the frequency. For $k=0$ the quasi-steady solution is derived. The transformation of the unsteady aerodynamics to the time domain is performed by means of a rational function approximation (RFA). The AIC matrix is approximated using Roger's method, which is defined by [20]

$$
\begin{aligned}
& Q_{j j}\left(s^{\star}\right)=Q_{0, j j}+Q_{1, j j} s^{\star}+\sum_{i=1}^{n_{p}} Q_{L_{i}, j j} \frac{s^{\star} I}{s^{\star}+p_{i}} \\
& =Q_{0, j j}+\frac{2 U_{\infty}}{c_{r}} D_{L} s^{\star} \\
& +C_{L}\left(s^{\star} I-\frac{c_{r}}{2 U_{\infty}} A_{L}\right)^{-1} B_{L} s^{\star} \\
& A_{L}=\frac{2 U_{\infty}}{c_{r}} \operatorname{diag}\left(\left[\begin{array}{lll}
-p_{1} I & \ldots & -p_{n_{p}} I
\end{array}\right]\right) \\
& B_{L}=\left[\begin{array}{lll}
I & \ldots & I
\end{array}\right]^{T} \\
& C_{L}=\left[\begin{array}{lll}
Q_{L_{1}, j j} & \ldots & Q_{L_{n_{p}}, j j}
\end{array}\right] \\
& D_{L}=\frac{c_{r}}{2 U_{\infty}} Q_{1, j j},
\end{aligned}
$$

where $s^{\star}=i k$ is equivalent to the Laplace variable $s$ for the reduced frequency $k$. When multiplying Equation (15) with the downwash $w_{j}$ from the right side and performing an inverse Laplace transformation, it becomes

$$
\begin{aligned}
\Delta c_{p j} & =\mathcal{L}^{-1}\left\{Q_{j j}\left(s^{\star}\right) w_{j}\left(s^{\star}\right)\right\} \\
& =\underbrace{Q_{0, j j} w_{j}}_{\text {quasi-steady }}+\underbrace{\mathcal{L}^{-1}\left\{D_{L}+C_{L}\left(s I-A_{L}\right)^{-1} B_{L}\right\} \dot{w}_{j}}_{\text {unsteady }} .
\end{aligned}
$$

As can be seen, the quasi-steady aerodynamics depend solely on the downwash $w_{j}$ defined in Equation (12). In contrast, the unsteady aerodynamics depend on its derivative $\dot{w}_{j}$ in time. The unsteady aerodynamics can be represented in the time domain as a state space system, defined by

$$
\begin{aligned}
\dot{x}_{L} & =A_{L} x_{L}+B_{L} \dot{w}_{j} \\
\Delta c_{p j_{\text {unsteady }}} & =C_{L} x_{L}+D_{L} \dot{w}_{j} .
\end{aligned}
$$

Thus, the chosen approximation introduces the additional states $x_{L}$. They are called lag states and represent the lagging behaviour of the unsteady aerodynamics $[9,10]$.

\section{Integrated Model}

In Figure 3 the structural dynamics are affected by the aerodynamic loads $P_{g}^{\text {aero }}$. These can be expressed as

$$
P_{g}^{\text {aero }}=q_{\infty} T_{k g}^{T} S_{k j} Q_{j j} w_{j}+q_{\infty} T_{a g}^{T} S_{r} c_{D},
$$

where the first term is affected by the downwash $w_{j}$ and the second term represents the aerodynamic drag loads with reference area $S_{r}$ and the transformation matrix from the mean aerodynamic centre to the structural grid $T_{a g}^{T}$. Matrix $S_{k j}$ depicts an integration relating the pressure in the aerodynamic boxes at point $j$ with the forces at the aerodynamic grid points $k$. The forces at the aerodynamic grid points $k$ are then interpolated onto the structural grid points via the transpose of the spline matrix $T_{k g}$. The splining model of the wing is exemplary shown in Figure 7 . Multiplication

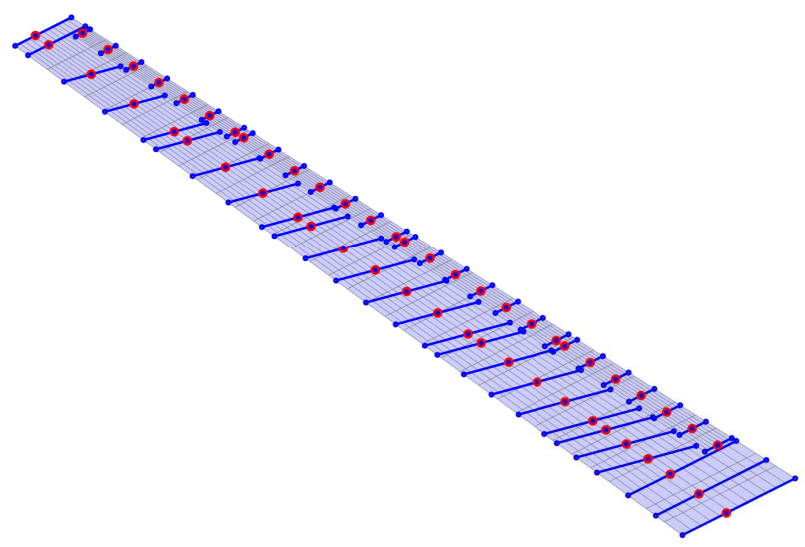

Figure 7: Splining between the aerodynamic model and structural model of the right wing [9]

with the dynamic pressure $q_{\infty}$ leads to the aerodynamic loads affecting the aircraft structure. To distinguish between the distributions of the aerodynamic loads with respect to the rigid and flexible body dynamics, Equation (19) is multiplied by $\Phi_{g b}^{T}$ and $\Phi_{g f}^{T}$ leading to

$$
\left[\begin{array}{l}
P_{b}^{\text {aero }} \\
P_{f}^{\text {aero }}
\end{array}\right]=\left[\begin{array}{l}
\Phi_{g b}^{T} \\
\Phi_{g f}^{T}
\end{array}\right] P_{g}^{\text {aero }} .
$$

The aerodynamic loads cause rigid and flexible body motions of the aircraft which, in turn, affect the aircraft aerodynamics. Therefore, the aeroelastic model is considered a loop between structural dynamics and aerodynamics $[9,10,12]$.

\section{Flight Testing}

The demonstrator aircraft, shown in Figure 8, is a jet-enginepowered UAV with $65 \mathrm{~kg}$ take-off mass and $7.1 \mathrm{~m}$ wingspan. It is flown manually by a pilot within visual line of sight. In rate control flight mode the surface deflections are directly linked to the joystick positions on the transmitter. The autopilot is used only during some test sequences, but not during take-off or landing. The geometry of the aircraft is summarized in Table 1. In Ref. 18 the aircraft design process of the demonstrator aircraft is described in more detail.

\section{Instrumentation of the Demonstrator}

The aircraft is equipped with integrated measurement equipment. The usual air data, position and inertial parameters 


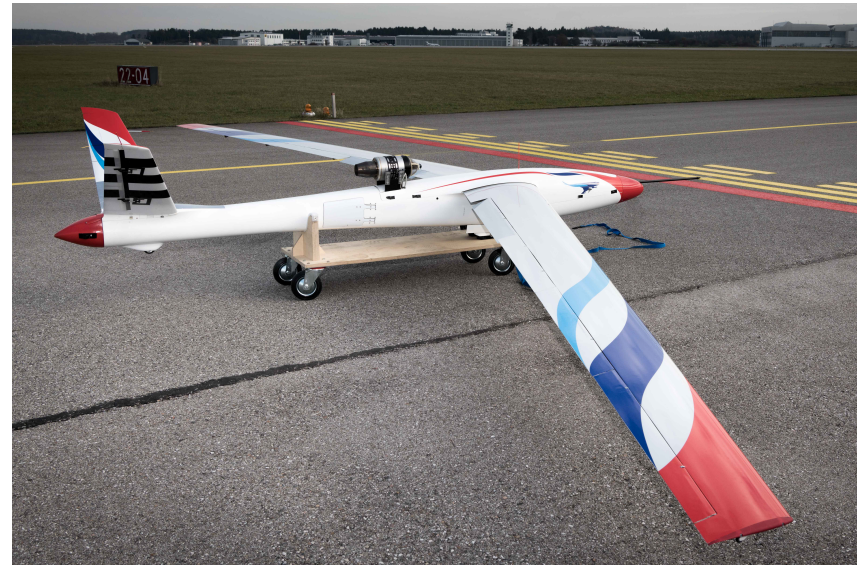

Figure 8: FLEXOP Aircraft before a flight test CDLR

Table 1: Geometry of the FLEXOP UAV

\begin{tabular}{l|l} 
Wing & $7.07 \mathrm{~m}$ \\
\hline Span: & $2.53 \mathrm{~m}^{2}$ \\
Area: & 19.74 \\
Aspect ratio: & $-0.52^{\circ}$ \\
Incidence: & $18.36^{\circ}$ \\
1/4-chord sweep: & 0.5 \\
Taper ratio: & $-2^{\circ}$ \\
Twist: & 8 \\
Number of control surfaces: & 8 \\
\hline \hline Fuselage & $3.42 \mathrm{~m}$ \\
\hline Length: & $0.315 \mathrm{~m}$ \\
Maximum height: & $0.3 \mathrm{~m}$ \\
Maximum width: & $1.27 \mathrm{~m}$ \\
\hline \hline Tail & $0.39 \mathrm{~m}^{2}$ \\
\hline Projected span: & 4.2 \\
Area: & $-4.33^{\circ}$ \\
Aspect ratio: & $19.83^{\circ}$ \\
Incidence: & 0.52 \\
1/4-chord sweep: & $35^{\circ}$ \\
Taper ratio: & Dihedral: \\
Number of control surfaces: & 4 \\
\hline \hline
\end{tabular}

are being logged on the aircraft. In addition, the wings are equipped with multiple IMUs spaced along the wing leading and trailing edge for vibration measurements. Figure 9 depicts the position of the IMUs. There are three telemetry systems installed onboard the aircraft:

1. the Unilog Link which sends the airspeed directly to the transmitter of the pilot,

2. the MAVLink which sends the data related to the flight state of the aircraft to the ground control station (GCS),

3 . and the Engineering Data Link which is concerned with structural and safety information regarding the aircraft.

The take-off weight of the configuration was kept at $65 \mathrm{~kg}$ for all the flights. For the first flight it was decided to shift the centre of gravity (CG) forwards in order to decrease the possibility of pitch-up stall. As the aircraft appeared to be well controllable in this configuration during the first flight, it was decided to keep the CG location fixed for all flights.

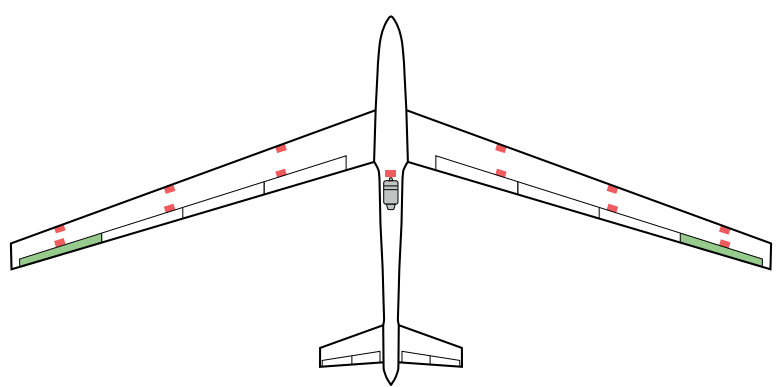

Figure 9: Location of the IMUs (red) [21]

\section{Environment}

The aircraft was flown at the Airport Oberpfaffenhofen (EDMO) [22], located around $20 \mathrm{~km}$ Southwest of Munich, Germany. During a flight test, the airspace of the airport was closed for 30 minutes, within which the flights with the demonstrator could take place. The airport has a $2286 \mathrm{~m}$ long concrete runway that is $45 \mathrm{~m}$ wide. Two sectors with different altitude limits are assigned for the flights by the traffic control.

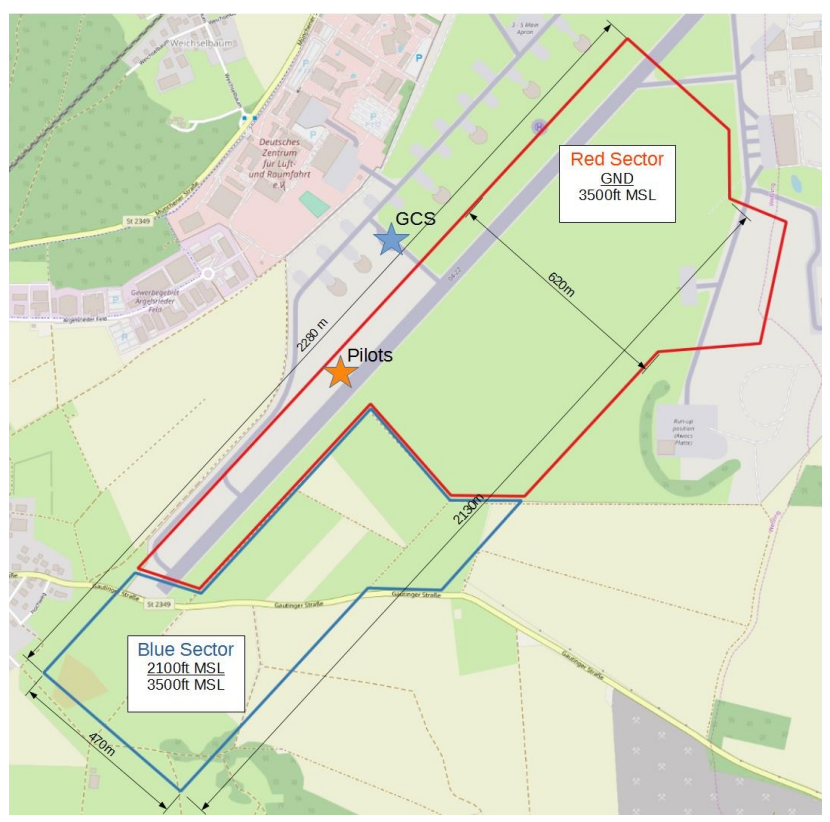

Figure 10: Allowed airspace for flight testing at EDMO airport

\section{Operations}

The core flight test crew consists of 5 people: the Flight Test Manager, the Flight Test Operator, the Flight Test Engineer, the Pilot-in-Command and the Back-up Pilot.

Usually the preparations for a flight test would begin with packing the entire equipment according to a checklist one day before the flight test. On the day of the flight test, the aircraft is assembled in the hangar at EDMO. Subsequently, the systems are checked. If the systems work properly, a preflight test briefing with the flight test crew follows. The flight test plan is discussed in detail.

After the pre-flight briefing, the aircraft, the ground control 


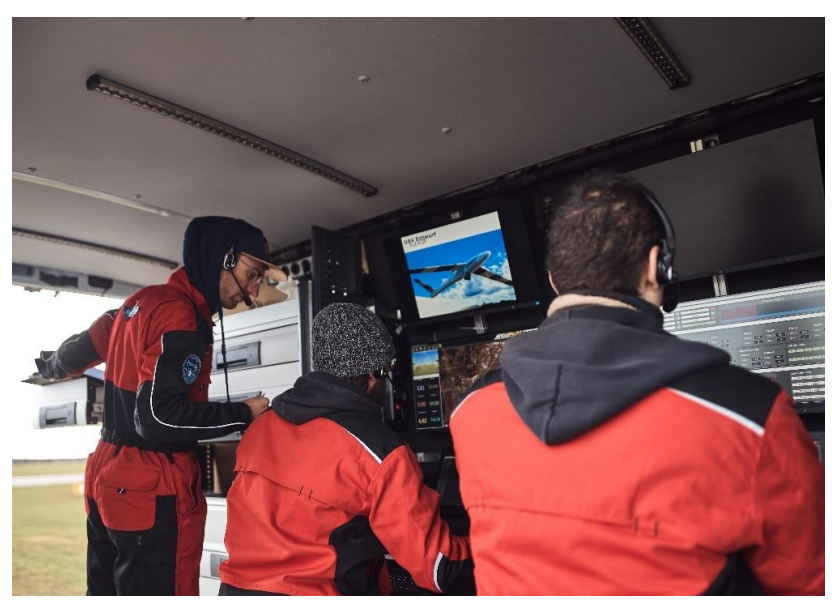

Figure 11: The Flight Test Manager (left), the Flight Test Operator (middle) and the Flight Test Engineer (right) inside the GCS during a flight test (F. Vogl/TUM)

station and the flight test crew moves to the nearest taxiway and prepares for a system start. A system check and engine start-up follows. If the start-up procedure was successful and clearance has been given by the EDMO tower, the aircraft is moved to the runway. The engine is started and the flight test takes place.

Subsequent to the flight test the aircraft is moved from the runway and a post-flight debriefing takes place. Important flight test and preparation items are noted down. If necessary, sensor $\operatorname{logs}$ and video footage are analysed in order to identify critical situations and implement mitigation strategies by revision of routines and procedures.

\section{Test Programme}

During the aircraft design stage it had to be ensured that the available airspace is enough to reach the flutter speed and sustain it for some time. To maximize this time, a mission design study has been done in Ref. 18. The study resulted in a horse-race track with turns at the highest safety speed and acceleration, deceleration and test legs.

Before flight testing the demonstrator aircraft various ground tests were performed. This involved system tests, wing static tests, ground vibration tests (GVT) and pilot and crew training in a flight test simulation environment. The performed tests are described in Ref. 23.

After the successful completion of the ground tests, the flight test programme followed. The programme was split into four phases:

1. Phase, baseline wing: maiden flight, instrument calibration and core system tests

2. Phase, baseline wing: rigid aircraft dynamics identification, aeroelastic model identification

3. Phase, aeroelastically tailored wing: static and dynamic manoeuvres for aeroelastic model identification

4. Phase, flutter wing: static and dynamic manoeuvres for aeroelastic model identification

The first and second phases of the flight test campaign dealt with gathering data with a stiffer baseline wing. Static aeroelastic testing manoeuvres, aircraft identification and performance manoeuvres, as well as autopilot functionality checks were all done at least once. All of the aircraft core systems were tested and performed as expected. The flight test phases 3 and 4 will be part of upcoming research activities.

The focus in the upcoming sections lies solely on the second flight test phase with respect to the identification of the baseline wing configuration.

\section{Performed Manoeuvres}

The parameter estimation process strongly depends on the performed manoeuvres in flight, as they define how well the characteristics of the aircraft can be determined. In order to define suitable excitation signals, a priori knowledge on the model is used. However, this is conflicting as the accuracy of the examined model determines the quality of the model parameters to be estimated [11]. Nevertheless, under the assumption, that the chosen modelling process provides realistic results, this approach is considered applicable.

Flight Mechanical Manoeuvres-The first goal is to update the flight mechanical model. Therefore all contributions resulting from the aircraft flexibility are neglected. Besides, unsteady aerodynamic effects are ignored, as their contribution to the flight mechanical model is assumed to be small. As a result, for the aerodynamic load $P_{b}^{\text {aero }}$ on the right-hand side of the rigid body EOM in Equation (2) it is only accounted for the downwash $w_{j b 1}, w_{j x 0}$ and $w_{j x 1}$ yielding to

$$
\begin{aligned}
P_{b}^{\text {aero }}= & q_{\infty} \underbrace{\Phi_{g b}^{T} T_{k g}^{T} S_{k j} Q_{j j} \frac{c_{r}}{2} D_{j k, 2} \Phi_{k a}}_{D Q_{h, b 1}}\left[\begin{array}{c}
1 \\
\beta_{a} \\
\alpha_{a} \\
p_{a} / U_{\infty} \\
q_{a} / U_{\infty} \\
r_{a} / U_{\infty}
\end{array}\right] \\
& +q_{\infty} \underbrace{\Phi_{g b}^{T} T_{k g}^{T} S_{k j} Q_{j j} D_{j k, 1} \Phi_{k x}}_{D Q_{h, x 0}} u_{x} \\
& +q_{\infty} \underbrace{\Phi_{g b}^{T} T_{k g}^{T} S_{k j} Q_{j j} \frac{c_{r}}{2 U_{\infty}} D_{j k, 2} \Phi_{k x}}_{D Q_{h, x 1}} \dot{u}_{x} .
\end{aligned}
$$

It is assumed, that the correlation between the control surfaces and the aerodynamic load given by the matrices $D Q_{h, x 0}$ and $D Q_{h, x 1}$ is accurately predicted by the proposed model. The focus is on an update of the rigid body contribution gathered in matrix $D Q_{h, b 1}$. It is a $6 x 6$-matrix with the entries

$$
D Q_{h, b 1}=\left[\begin{array}{cccccc}
0 & 0 & 0 & 0 & 0 & 0 \\
0 & f_{y \beta} & 0 & f_{y p} & 0 & f_{y r} \\
f_{z 0} & 0 & f_{z \alpha} & 0 & f_{z q} & 0 \\
0 & m_{x \beta} & 0 & m_{x p} & 0 & m_{x r} \\
m_{y 0} & 0 & m_{y \alpha} & 0 & m_{y q} & 0 \\
0 & m_{z \beta} & 0 & m_{z p} & 0 & m_{z r}
\end{array}\right] .
$$

There are 15 parameters, that are to be estimated. As can be seen the parameters related to forces in $x$-direction are neglected, due to the mentioned constraints of the model. Many more entries are equal to zero or considered too small to have a significant influence on the parameter estimation. The remaining parameters can be associated with either a longitudinal or a lateral aircraft motion.

In Table 2 the performed manoeuvres, separated in longitudinal and lateral, are listed with the parameters, that mainly contribute to the aircraft motion. The definition of the excitation signals for the short period, phugoid and dutch-roll mode are determined based on an a priori analysis of the initial model. The phugoid is excited by an elevator pulse, that is chosen to last 2 seconds with an amplitude of approximately 
Table 2: Performed manoeuvres for the parameter estimation [11]

Longitudinal

\begin{tabular}{l|l}
\hline Steady level flight: & $f_{z 0}, f_{z \alpha}, m_{y 0}, m_{y \alpha}$ \\
Pushover-pullup: & $f_{z 0}, f_{z \alpha}, f_{z q}$ \\
Short period: & $f_{z \alpha}, f_{z q}, m_{y \alpha}, m_{y q}$ \\
Phugoid: & $f_{z 0}, f_{z \alpha}, f_{z q}, m_{y 0}, m_{y \alpha}, m_{y q}$ \\
\hline \hline Lateral & \multicolumn{2}{|l}{} \\
\hline Steady sideslip: & $f_{y \beta}, m_{z \beta}$ \\
Dutch-roll: & $f_{y \beta}, f_{y r}, m_{x \beta}, m_{x r}, m_{z \beta}, m_{z r}$ \\
Bank-to-bank: & $f_{y p}, m_{x p}, m_{z p}$ \\
\hline \hline
\end{tabular}

$3^{\circ}$. This elevator deflection was found to be appropriate to excite the phugoid mode of the aircraft [11]. The dutchroll mode can be excited by a doublet on the rudder. The amplitude is chosen to be around $3^{\circ}$, while the half time length $\Delta t_{\text {doublet }}$ of the doublet is calculated with the dutchroll frequency $\omega_{\text {dutch-roll }}$ by the rule of thumb

$$
\Delta t_{\text {doublet }} \approx \frac{2.3}{\omega_{\text {dutch-roll }}}
$$

to be 1.22 seconds [11]. The dutch-roll frequency $\omega_{\text {dutch-roll }}$ is determined from the simulation in advance of the flight test. Equivalently, the short period mode can be observed by exciting the elevator with a doublet. Equation (23) gives a $\Delta t_{\text {doublet }}$ of 0.24 seconds with the pre-determined frequency

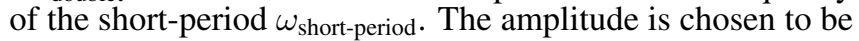
around $6^{\circ}$. The steady level flight, pushover-pullup, steady sideslip and bank-to-bank manoeuvres were flown manually by the pilot.

Excitation of Flexible Modes-The flexible properties of the demonstrator aircraft were tested in a static test and GVT [24]. The results are used to update the structural model matrices $K_{f f}$ and $B_{f f}$ introduced in Equation (3). However, in flight the occurring aeroelastic modes caused by the flexibility of the aircraft might vary from the model prediction. Therefore, the aeroelastic modes of the demonstrator aircraft in flight are analysed based on readings of the wing IMUs. A flight test lag of 12 seconds is considered for that.

\section{UPdATE OF THE Rigid Body MOdEL}

When it comes to updating an aircraft model or rather specific model parameters, a suitable process needs to be set up. On the one hand a model structure must be given including parameters to be estimated and on the other hand an optimization algorithm to find the somewhat best model parameters needs to be given. There exist different optimization algorithms to estimate model parameters, like the output error method (OEM), the filter error method (FEM) and more. For an description of different optimization algorithms it is referred to Ref. 11,25. Within the scope of this paper the OEM based on the maximum likelihood estimation is chosen.

\section{Output Error Method}

In Figure 12 the basic procedure of the OEM is shown. The upper path represents the flight test, where the outcome is the measured inputs and outputs. The OEM assumes, that the outputs are affected by measurement noise. Process noise, however, is neglected. Subsequently, the inputs are fed into the mathematical model to conduct a simulation of the considered flight test manoeuvre. Based on the difference

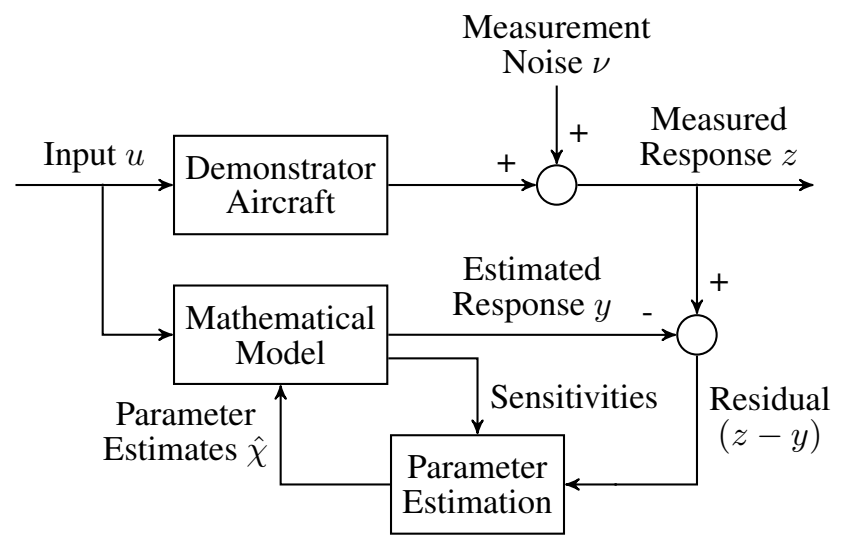

Figure 12: Procedure of the OEM [25]

between the flight test measurements and the simulation outputs, the parameters of the mathematical model are updated by means of an optimisation [11,25].

It is assumed, that the model equations are given in the form of

$$
\begin{aligned}
\dot{x}(t) & =f(x(t), u(t), \chi), x\left(t_{0}\right)=x_{0} \\
y(t) & =g(x(t), u(t), \chi) \\
z\left(t_{k}\right) & =y\left(t_{k}\right)+\nu\left(t_{k}\right) .
\end{aligned}
$$

The first two equations describe the proposed mathematical model. They dependent on the desired parameters $\chi$. The last equation provides the relation between the discrete flight test measurements $z$ and the output of the measurement equation $y$ at a time instant $t_{k}$. They exclusively differ in the measurement noise $\nu$. The noise is considered stochastic and is characterized by Gaussian white noise with zero mean. Its definition is

$$
\begin{aligned}
E\left\{\nu\left(t_{k}\right)\right\} & =0 \\
E\left\{\nu\left(t_{k}\right) \nu\left(t_{l}\right)^{T}\right\} & =R \delta_{k l} .
\end{aligned}
$$

The second expression of Equation (25) suggests that the noise represents white noise, as it is time independent. Simultaneously the amplitude depends on chance defined by a Gaussian distribution with covariance matrix $R$. As a result the measurement vector $z\left(t_{k}\right)$ with dimension $n_{z}$ is affected by Gaussian white noise and therefore its values are assumed to be Gaussian distributed with the probability density function

$$
\begin{aligned}
& p\left(z\left(t_{k}\right) \mid \chi\right)=\frac{1}{(2 \pi)^{\left(n_{z} / 2\right)} \sqrt{|R|}} \\
& \exp \left(-\frac{1}{2}\left(z\left(t_{k}\right)-y\left(t_{k}\right)\right)^{T} R^{-1}\left(z\left(t_{k}\right)-y\left(t_{k}\right)\right)\right) .
\end{aligned}
$$

With respect to Equation (24) the expected value of $z\left(t_{k}\right)$ is assumed to be $E\left\{z\left(t_{k}\right)\right\}=y\left(t_{k}\right)$ for the model parameters $\chi$. 
For a set of $N$ measurements the likelihood function becomes

$$
\begin{aligned}
& p\left(z\left(t_{1}\right), \ldots, z\left(t_{N}\right) \mid \chi\right)=\prod_{k=1}^{N} p\left(z\left(t_{k}\right) \mid \chi\right) \\
&=\left[(2 \pi)^{n_{z}}|R|\right]^{-N / 2} \\
& \exp \left(-\frac{1}{2} \sum_{k=1}^{N}\left(z\left(t_{k}\right)-y\left(t_{k}\right)\right)^{T} R^{-1}\left(z\left(t_{k}\right)-y\left(t_{k}\right)\right)\right) .
\end{aligned}
$$

Goal of the maximum likelihood method (MLM) is to identify the model parameters $\chi$, which maximise the probability defined by Equation (27). The optimal solution is the maximum likelihood estimate obtained as

$$
\begin{aligned}
\hat{\chi}_{M L} & =\arg \left\{\max _{\chi} p(z \mid \chi)\right\} \\
& =\arg \left\{\min _{\chi}(-\ln p(z \mid \chi))\right\} .
\end{aligned}
$$

For greater ease of handling the negative logarithm of the likelihood function $p(z \mid \chi)$ is considered, which simplifies Equation (27) to the cost function

$$
\begin{aligned}
J(\chi, R)= & \frac{1}{2} \sum_{k=1}^{N}\left(\left(z\left(t_{k}\right)-y\left(t_{k}\right)\right)^{T} R^{-1}\left(z\left(t_{k}\right)-y\left(t_{k}\right)\right)\right) \\
& +\frac{N}{2} \ln (|R|)+\frac{N n_{z}}{2} \ln (2 \pi) .
\end{aligned}
$$

At this point it is assumed, that the covariance matrix $R$ is unknown a priori. As $R$ depends on the model parameters $\chi$ and vice versa, the relaxation strategy is used to find the optimal solution of the redefined likelihood function (29) in two steps. Firstly, for a given parameter vector $\chi$ the maximum likelihood estimate of $R$ is obtained by setting the partial derivative $\partial J(\chi, R) / \partial R$ to zero. This yields

$$
R=\frac{1}{N} \sum_{k=1}^{N}\left(z\left(t_{k}\right)-y\left(t_{k}\right)\right)\left(z\left(t_{k}\right)-y\left(t_{k}\right)\right)^{T} .
$$

Secondly, substituting (30) in (29) provides

$$
J(\chi)=\frac{1}{2} n_{z} N+\frac{N}{2} \ln (|R|)+\frac{N n_{z}}{2} \ln (2 \pi) .
$$

Apart from $\ln (|R|)$ all terms in Equation (31) are independent from the model parameters $\chi$. The cost function therefore reduces to

$$
J(\chi)=|R| .
$$

Equation (32) is solved iteratively for the optimal model parameter $\chi$ by means of a Gauss-Newton algorithm. Ref. 11 provides a deeper insight in the optimisation process.

\section{Two-Step Method}

By means of the two-step method (TSM) the model parameters can be determined. The TSM divides the state and parameter estimation problem in a flight path reconstruction (FPR) and a parameter identification part. The FPR is used to accurately reconstruct the time history of the aircraft states during the manoeuvre and besides allows the determination of potential instrumentation errors. As some sensor readings, like the angle of attack and the airspeed, are prone to be inaccurate, the measurements are improved based on past, present and future data and the flight mechanical equations. Subsequently, the identification of the model parameters follows $[11,26]$. The success of the TSM depends on the aircraft to be tested, the aircraft instrumentation, the excitation signals, the mathematical model selected for identification and the chosen algorithm for the analysis and adaption of the model [26]

Flight Path Reconstruction-The FPR is based on a nonlinear state-space system consisting of flight mechanical state and measurement equations. The considered inputs are the translational accelerations $a_{b m}$ and the rotational rates $\Omega_{b m}$ measured in flight by an IMU placed in the fuselage. The states are the velocity vector $V_{b}$, the Euler angles $\phi, \theta$ and $\psi$ and the altitude $h$. The resulting state equations are given by

$$
\begin{aligned}
\dot{V}_{b} & =a_{b}-\left(\Omega_{b, m}-\Delta \Omega_{b}\right) \times V_{b}+T_{b e} g_{e} \\
{\left[\begin{array}{c}
\dot{\phi} \\
\dot{\theta} \\
\dot{\psi}
\end{array}\right] } & =\left[\begin{array}{ccc}
1 & \sin \phi \tan \theta & \cos \phi \tan \theta \\
0 & \cos \phi & -\sin \phi \\
0 & \frac{\sin \phi}{\sin \theta} & \frac{\cos \phi}{\sin \theta}
\end{array}\right]\left(\Omega_{b, m}-\Delta \Omega_{b}\right) \\
\dot{h} & =\left[\begin{array}{lll}
0 & 0 & -1
\end{array}\right] T_{b e}^{-1} V_{b}
\end{aligned}
$$

Starting point of the state equations is the equilibrium of forces of the rigid body equation of motion. Solving Equation (2) for $\dot{V}_{b}$ leads to Equation (33), where $\Omega_{b}$ is replaced by its flight test measurement $\Omega_{b, m}$ including a potential sensor bias $\Delta \Omega_{b}$. The translational acceleration $a_{b}$ is given with respect to the center of gravity [11]. It is determined by

$$
\begin{aligned}
a_{b}= & a_{b, m}-\dot{\Omega}_{b} \times d_{s} \\
& -\left(\Omega_{b, m}-\Delta \Omega_{b}\right) \times\left(\left(\Omega_{b, m}-\Delta \Omega_{b}\right) \times d_{s}\right)-\Delta a_{b} .
\end{aligned}
$$

The acceleration measurement $a_{b, m}$ needs to be corrected for the coriolis and the centrifugal force caused by the offset between the acceleration sensor position and the center of gravity $d_{s}$. A potential sensor bias is covered by $\Delta a_{b}$. Additional state equations of the Euler angles $\phi, \theta, \psi$ are considered through Equation (34). The remaining state equation is given by Equation (35). The inverse of $T_{b e}$ transforms the velocity $V_{b}$ to the Earth-fixed frame of reference. Extracting only the element, which contributes to the z-direction, and changing the sign leads to the derivative of the altitude $\dot{h}$ [11].

The outputs or reconstructed instrumentation measurements are the true airspeed $U_{\infty, r}$, the angle of attack $\alpha_{r}$, the sideslip angle $\beta_{r}$, the Euler angles $\phi_{r}, \theta_{r}$ and $\psi_{r}$ and the altitude $h_{r}$. The corresponding measurement equations are given by

$$
\begin{aligned}
U_{\infty, r} & =\left\|V_{b}\right\|_{2} \\
\alpha_{r} & =K_{\alpha} \tan ^{-1}\left(\frac{V_{n b, z}}{V_{n b, x}}\right)+\Delta \alpha \\
\beta_{r} & =K_{\beta} \sin ^{-1}\left(\frac{V_{n b, y}}{\left|V_{n b}\right|}\right)+\Delta \beta \\
\phi_{r} & =\phi \\
\theta_{r} & =\theta \\
\psi_{r} & =\psi \\
h_{r} & =h .
\end{aligned}
$$


As the $\alpha$ and $\beta$ measurements of the noseboom are sensitive to errors, the scaling and bias variables $K_{\alpha}, \Delta \alpha, K_{\beta}$ and $\Delta \beta$ are introduced. The velocity vector $V_{n b}$ at the noseboom is determined by

$$
V_{n b}=V_{b}+\left(\Omega_{b, m}-\Delta \Omega_{b}\right) \times d_{n b},
$$

where $d_{n b}$ is the distance between the aircraft CG and the noseboom.

The unknown parameters $\Delta \Omega_{b}, \Delta a_{b}, K_{\alpha}, \Delta \alpha, K_{\beta}, \Delta \beta$ as well as the initial states $V_{b 0},\left[\phi_{0} \theta_{0} \psi_{0}\right]^{T}, h_{0}$ of Equations (33)-(35) are determined based on the OEM algorithm. It is assumed that the difference between the flight test measurements and the reconstructed measurements in (37)-(43) is only coming from the measurement noise $\nu$. The residual $(z-y)$, which is equal to $\nu$, is therefore to be minimized [11].

The FPR is performed for each considered manoeuvre type separately. Figures 13 and 14 depict the FPR results exemplary for a pushover-pullup manoeuvre (POPU) and for a sideslip manoeuvre (SL) in comparison with the measured flight test data (FTD). Only the measurement variables that
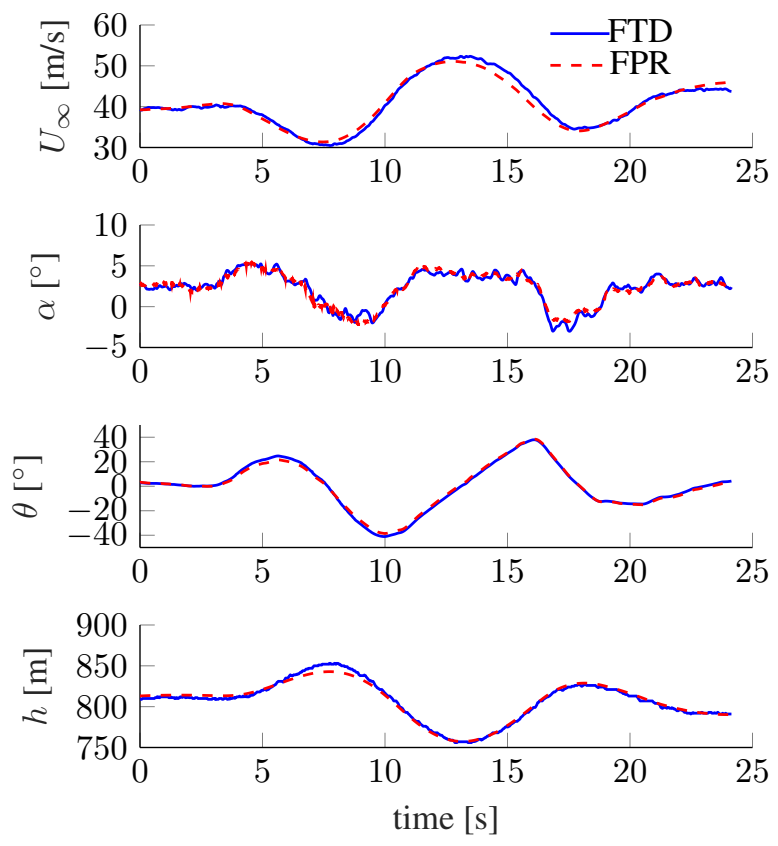

Figure 13: Comparison of reconstructed and flight test measurements (POPU)

play a major role for the manoeuvres are shown. For the POPU manoeuvre it can be seen, that $\alpha$ changes dynamically, while the remaining measurements are rather smooth. Nevertheless, the reconstructed $\alpha$ follows very closely the measurement.

The sideslip manoeuvre is not performed at a constant $\beta$ as intended. However, it still offers the opportunity for updating lateral model parameters. The FPR follows the trends of the observations very well. An exception is the reconstructed true airspeed $U_{\infty}$ which follows the trend of the measurement, but does not change as dynamically. As this is behaviour is not observed for the additional measurements, it is valid to say the true airspeed is more strongly affected by disturbance for the considered sideslip manoeuvre.

Parameter Estimation-The parameter estimation is the second step of the TSM. The control surface deflections commanded during the various flight test manoeuvres are fed in
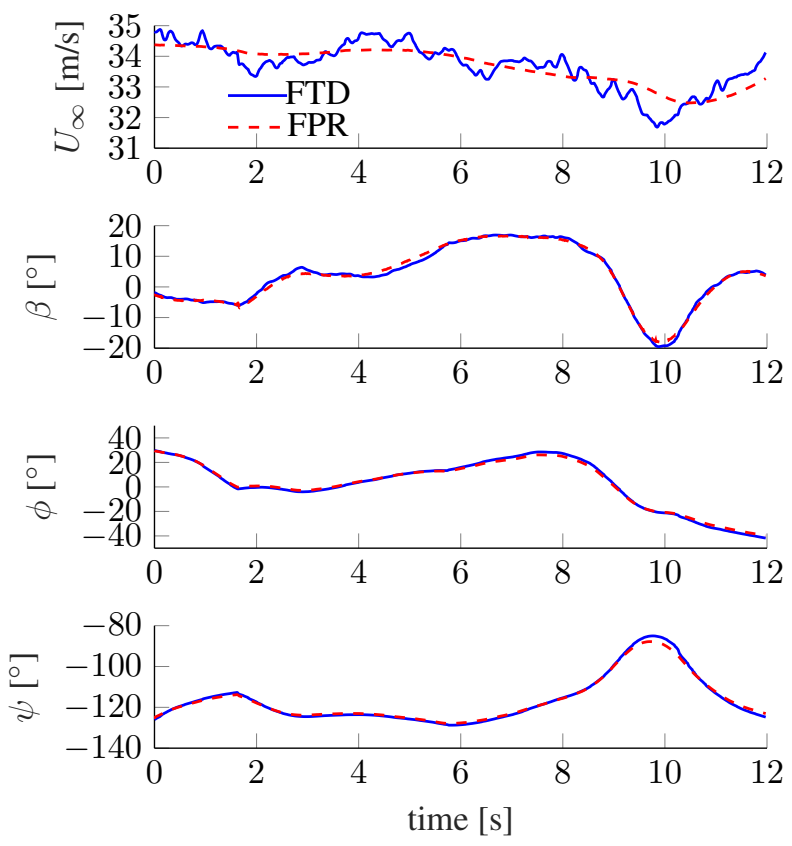

Figure 14: Comparison of reconstructed and flight test measurements (SL)

the rigid body equation of motion (2). As mentioned before the parameters of the matrix $D Q_{h, b 1}$ defined in Equation (22) are to be estimated. Based on the comparison between the outputs of the flight test $z$ and the simulation $y$ the model parameters are updated like described in the section Output Error Method.

The parameters corresponding to the longitudinal and lateral motion respectively are updated in separate steps. At first, the lateral manoeuvres are used to improve the matrix $D Q_{h, b 1}$ with respect to the parameters $f_{y \beta}, f_{y p}, f_{y r}, m_{x \beta}, m_{x p}$, $m_{x r}, m_{z \beta}, m_{z p}$ and $m_{z r}$. Subsequently, the longitudinal parameters $f_{z 0}, f_{z \alpha}, f_{z q}, m_{y 0}, m_{y \alpha}$ and $m_{y q}$ are updated with the matrix $D Q_{h, b 1}$ coming from the previous step. The final step is to redo the lateral update. This approach is chosen, because the longitudinal manoeuvres also feature lateral contributions and vice versa. Therefore, a strict separation of the manoeuvres is not possible.

At the end, the OEM leads to the parameters summarized in Tables 3 and 4 . Of note is that the $f_{z 0}$ contributing to

Table 3: Initial and final longitudinal parameters

\begin{tabular}{|c|c|c|}
\hline er & itial & Fina \\
\hline$f_{z 0}:$ & & \\
\hline$f_{z \alpha}:$ & -1 & 19 \\
\hline$f_{z q}:$ & -2 & 021 \\
\hline$m_{y 0}:$ & 0.0 & - \\
\hline$m$ & -1 . & -9 \\
\hline$m_{y q}:$ & -5.06 & -2.21 \\
\hline
\end{tabular}

the lift with respect to camber and drag was lightly overestimated with the CFD calculations mentioned before. The corresponding moment coefficient $m_{y 0}$, however, undergoes a relatively big change and switches sign. The $f_{z \alpha}$ and $f_{y \beta}$ values do not change much, which proves the validity of the VLM/DLM modelling approach with respect to linear trends. There are further parameters, that differ strongly from their 
Table 4: Initial and final lateral parameters

\begin{tabular}{l|l|l}
\multicolumn{1}{c}{ Parameter } & \multicolumn{1}{l}{ Initial } & \multicolumn{1}{l}{ Final } \\
\hline$f_{y \beta}:$ & -0.621 & -0.661 \\
$f_{y p}:$ & -0.12 & -0.876 \\
$f_{y r}:$ & 0.94 & 1.27 \\
$m_{x \beta}:$ & -0.382 & -2.08 \\
$m_{x p}:$ & -40.97 & -47.13 \\
$m_{x r}:$ & 0.477 & 12.16 \\
$m_{z \beta}:$ & 0.464 & 0.48 \\
$m_{z p}:$ & 0.11 & -1.47 \\
$m_{z r}:$ & -1.5 & -1.51 \\
\hline \hline
\end{tabular}

initial values. It is still under investigation, what the reason is.

When the pushover-pullup (POPU) manoeuvre is performed with the model featuring the estimated parameters (PE), one can recognize a strong similarity with the reconstructed flight test data (FPR). Figure 15 depicts the trend of some of the observation variables affected by a longitudinal motion. The
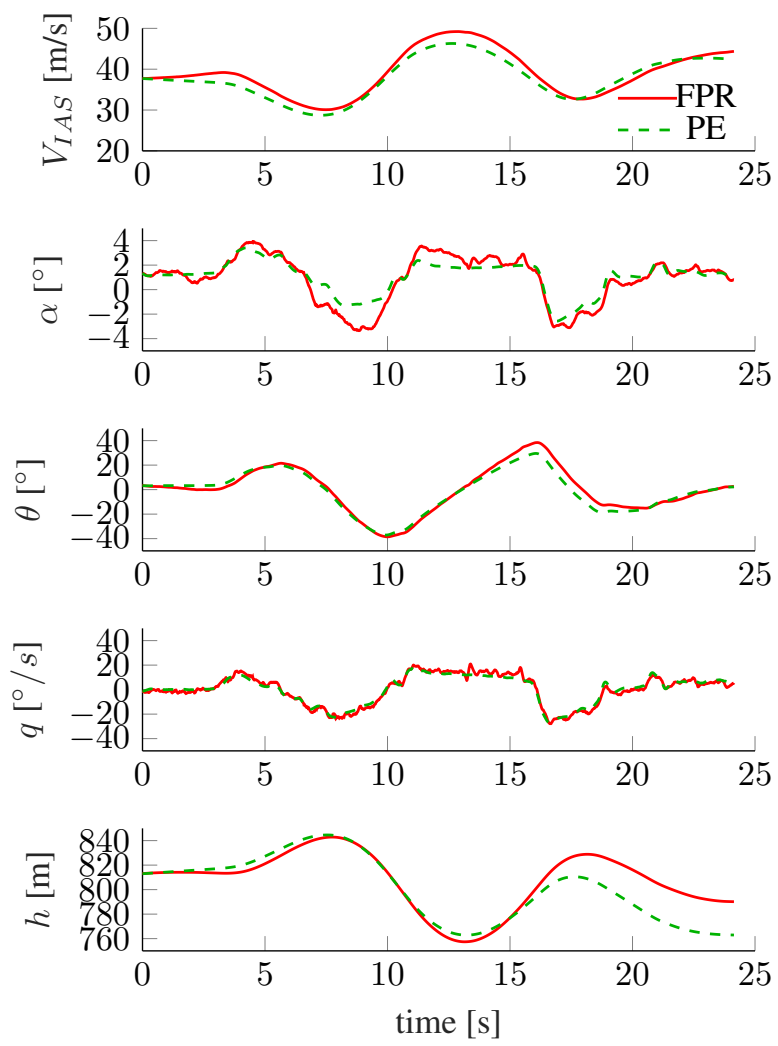

Figure 15: Comparison between reconstructed and simulated measurements (POPU)

difference between the reconstructed and simulated angle of attack $\alpha$ reveals a higher sensitivity to disturbances. However, especially the pitch rate $q$ matches very well between both data sets.

The measurements of the sideslip manoeuvre exhibited in Figure (16) proves, that the set of estimated parameters of the model fits well with the flight test data.
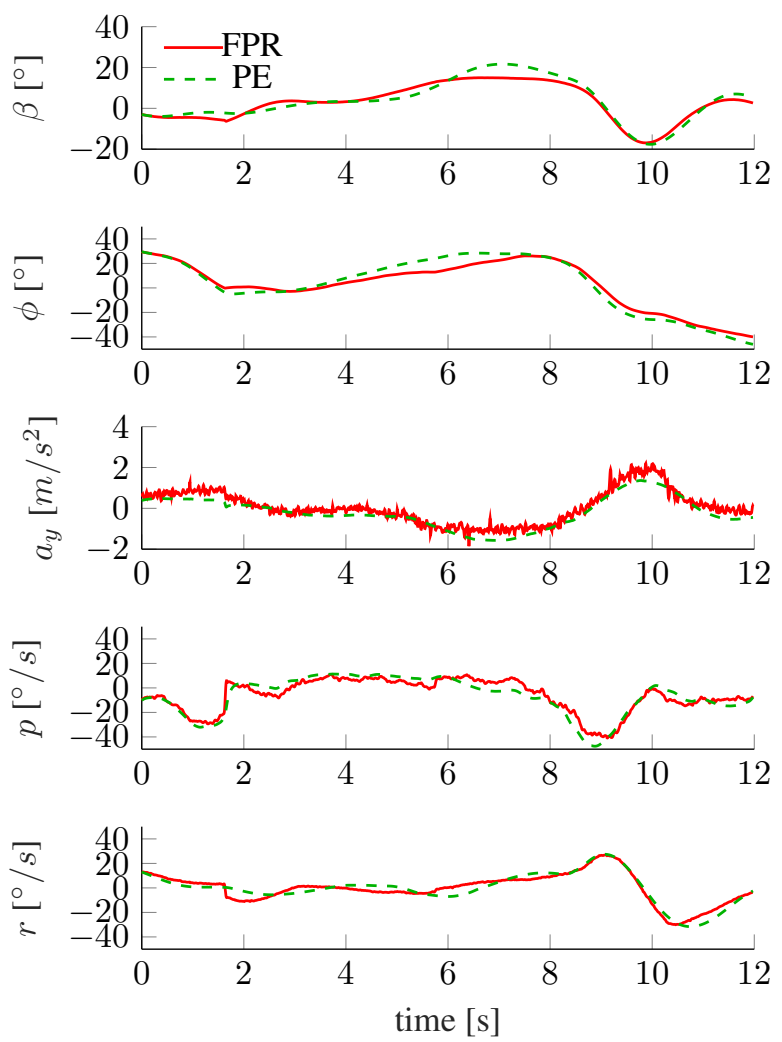

Figure 16: Comparison between reconstructed and simulated measurements (SL)

\section{FleXible Mode IdENTIFICATION}

This section primarily concerns the identification of the flexible aircraft dynamics of a highly flexible flutter demonstrator aircraft based on in-flight data. The analysis of in-flight data poses a number of specific challenges that are related to the complex test structure and the difficulties caused by test conditions. Therefore, an automatically running robust Stochastic Subspace Identification (rSSI) method is introduced, which only needs the structural dynamic response data of the aircraft due to non-deterministic natural and/or operational excitations which are provided by atmospheric turbulence and/or pilot control inputs for determination of the stochastic system matrices and consequently of the flight modal parameters. In this method, as the only available information are the measured responses, its assumed that the aircraft are excited by white noise processes and that the responses are realization of these processes. The flexible aircraft dynamics are described by flight modal data, which include eigenfrequencies, damping ratios and eigenvectors of the aircraft during a specified flight condition. The experimental flight modal data obtained by this methodology are then compared with the predicted results supplied by the numerical model by means of modal correlation analysis for the quality assessment of the actual numerical model of the flexible aircraft.

Within the framework of the FliPASED project, a robust Stochastic Subspace Identification (rSSI) method in timedomain has been implemented for identification of flight modal paramerters, which are often used for quality assessment of the numerical model and consequently its validation with respect to flexible aircraft dynamics. In case of sub- 
stantial discrepancies between predictions of the model and those yielded by experimental data from tests on the aircraft structure, the numerical aircraft model has to be updated until model predictions and experimental results have a sufficient correlation. At this point it is important to note that this section of the paper solely addresses the rSSI algoritm and its application to the in-flight data with a subsequent modal correlation analysis between results from numerical and flight test domain.

\section{Data-driven Stochastic System Identification}

SSI techniques are examples of the recent advances in modal parameter estimation methods in time-domain. These methods have become very popular due to their robustness and precision verified even in the presence of highly noisy data. In these methods, as the only available information are the outputs, it is assumed that the systems (here the tested aircraft structure) are excited by white noise processes and that the outputs (measured responses) are realization of these processes [27]. In the following, a detailed description of a robust data-driven SSI (rSSI-DATA) method is presented. One of the major advantages of this identification method is that it enables the extraction of modal parameters directly from the time data which results in a reduction of additional preprocessing time compared to its covariance-driven (SSICOV) counterpart. We start with state-space respresentation of combined deterministic-stochastic systems. For these systems, both the known input $u_{k}$ and the process and measurement noise $w_{k}$ and $v_{k}$ are different from zero. We will consider state space models of the form:

$$
\begin{aligned}
x_{k+1} & =A x_{k}+B u_{k}+w_{k} \\
y_{k} & =C x_{k}+D u_{k}+v_{k}
\end{aligned}
$$

with $w_{k}$ and $v_{k}$ zero mean, white vector sequences with covariance matrix:

$$
\mathbf{E}\left[\left(\begin{array}{c}
w_{p} \\
v_{p}
\end{array}\right)\left(\begin{array}{ll}
w_{p}^{T} & v_{p}^{T}
\end{array}\right)\right]=\left(\begin{array}{cc}
Q & S \\
S^{T} & P
\end{array}\right) \delta_{\mathbf{p q}} .
$$

where $\mathbf{E}[\cdot]$ is the expected value operator and $\delta_{p q}$ the Kronecker delta. Since $w_{k}$ and $v_{k}$ are zero mean white noise vectors sequences, independent of $x_{k}$, it follows:

$$
\begin{aligned}
\mathbf{E}\left[\mathbf{x}_{\mathbf{k}} \mathbf{v}_{\mathbf{k}}^{\mathbf{T}}\right] & =0, \\
\mathbf{E}\left[\mathbf{x}_{\mathbf{k}} \mathbf{w}_{\mathbf{k}}^{\mathbf{T}}\right] & =0 .
\end{aligned}
$$

The vectors $y_{k} \in R^{l}$ and $u_{k} \in R^{m}$ are measured (given) outputs and inputs of the system at the discrete time $k$ with $k=1,2, \ldots N_{s}$, where $N_{s}$ is the number of data samples. The order of the system $n$, system matrices $A \in R^{n x n}$, $B \in R^{n x m}, C \in R^{l x n}, D \in R^{l x m}$ and the covariance matrices $Q \stackrel{\in}{\in} R^{n x n}, S \in R^{n x l}, P \in R^{l x l}$ have to be determined. In our case of purely stochastic systems with no external inputs $\left(u_{k} \equiv 0\right)$ the state-space model from the Equation (45) becomes:

$$
\begin{aligned}
x_{k+1} & =A x_{k}+w_{k}, \\
y_{k} & =C x_{k}+v_{k}
\end{aligned}
$$

In the following sections a robust Stochastic Subspace Identification (rSSI) algorithm for systems described in the previous Equations (48) will be introduced.

\section{Robust Stochastic Subspace Identification Algorithm}

In this section a robust subspace algorithm for subspace identification will be introduced which can be applied for combined deterministic-stochastic systems, whereby we only consider the subspace identification of purely stochastic system which is relevant for our case. The stochastic identification problem addresses the determination of the stochastic system matrices from given output-only data. We make use of the RQ and the singular value decomposition (SVD). Before we start with the algorithm, we introduce the Block Hankel matrices which play a major role in subspace identification algorithms.

Block Hankel matrices - Hankel matrices can be defined from the measured input-output or only from output data in case of purely stochastic systems. Output block Hankel matrices are constructed as follows:

$Y_{1 \mid 2 i} \stackrel{\text { def }}{=}\left[\begin{array}{ccccc}y_{1} & y_{2} & y_{3} & \ldots & y_{j} \\ y_{2} & y_{3} & y_{4} & \ldots & y_{j+1} \\ \ldots & \ldots & \ldots & \ldots & \ldots \\ y_{i} & y_{i+1} & y_{i+2} & \ldots & y_{i+j-1} \\ \hline y_{i+1} & y_{i+2} & y_{i+3} & \ldots & y_{i+j} \\ y_{i+2} & y_{i+3} & y_{i+4} & \ldots & y_{i+j+1} \\ \ldots & \ldots & \ldots & \ldots & \ldots \\ y_{2 i} & y_{2 i+1} & y_{2 i+2} & \ldots & y_{2 i+j-1}\end{array}\right]$

$\stackrel{\text { def }}{=}\left(\frac{Y_{1 \mid i}}{Y_{i+1 \mid 2 i}}\right) \stackrel{\text { def }}{=}\left(\frac{Y_{p}}{Y_{f}}\right)$

$\stackrel{\text { def }}{=}\left[\begin{array}{ccccc}y_{1} & y_{2} & y_{3} & \ldots & y_{j} \\ y_{2} & y_{3} & y_{4} & \ldots & y_{j+1} \\ \ldots & \ldots & \ldots & \ldots & \ldots \\ y_{i} & y_{i+1} & y_{i+2} & \ldots & y_{i+j-1} \\ y_{i+1} & y_{i+2} & y_{i+3} & \ldots & y_{i+j} \\ \hline y_{i+2} & y_{i+3} & y_{i+4} & \ldots & y_{i+j+1} \\ \ldots & \ldots & \ldots & \ldots & \ldots \\ y_{2 i} & y_{2 i+1} & y_{2 i+2} & \ldots & y_{2 i+j-1}\end{array}\right]$

$\stackrel{\text { def }}{=}\left(\frac{Y_{1 \mid i+1}}{Y_{i+2 \mid 2 i}}\right) \stackrel{\text { def }}{=}\left(\frac{Y_{p}^{+}}{Y_{f}^{-}}\right)$

where $i$ is used-defined parameter and denotes the number of block rows with $Y_{1 \mid 2 i} \in R^{2 l i x j}$. It should be chosen so that $i>2 N_{m}$, where $N_{m}$ represents the number of physical modes. The number of columns $j$ is mostly equal to $N_{s}-2 i+1$ in case of that all given data samples are used. The subscript $p$ denotes past and the subscript $f$ stands for future. The matrices $Y_{p}$ (the past outputs) and $Y_{f}$ (the future outputs) are defined by partitioning $Y_{1 \mid 2 i}$ into two equal parts of $i$ block rows. The matrices $Y_{p}^{+}$and $Y_{f}^{-}$are constructed by shifting the border between past and future one block row downward.

Extended observability matrix $\Gamma_{i}$-In subspace identification algorithms the observability matrix and its structure play a key role. The extended observability matrix $\Gamma_{i}(i>n)$ where the subscript $i$ denotes the number of block rows, is defined 
as follows:

$$
\Gamma_{i}=\left[\begin{array}{c}
C \\
C A \\
C A^{2} \\
\vdots \\
C A^{i-1}
\end{array}\right] \in R^{l i x n}
$$

The pair $\{A, C\}$ is assumed to be observable. The determination of the system matrices $A$ and $C$ can be realized by using the shift structure of the extended observability matrix $\Gamma_{i}$ which will be described in the next section.

Robust Algorithm and Numerical Implementation-The robust algorithm which will be described here only consider the subspace identification of purely stochastic systems which addresses the calculation of the stochastic system matrices from given output-only data.

Step 1. The robust algorithm starts with the $R Q$ decomposition of the block Hankel matrix formed of the output data:

$$
H=\frac{1}{\sqrt{j}} Y_{1 \mid 2 i}=\left(\begin{array}{l}
Y_{p} \\
Y_{f}
\end{array}\right)=R \cdot Q^{T} \in R^{2 l i x j}
$$

with the orthogonal matrix $Q^{T} \in R^{2 l i x j}$ and the lower triangular matrix $R \in R^{2 l i x 2 l i}$ [28].

For the calculation of the system matrices within the algorithm, only the $R$ factor of this decomposition is needed which results in reduction of computation time.

For convenience of notation, we partition the $R$ matrix as follows: $\quad l i \quad l \quad l(i-1)$

$$
R=\left[\begin{array}{ccc}
R_{1,1} & 0 & 0 \\
R_{2,1} & R_{2,2} & 0 \\
R_{3,1} & R_{3,2} & R_{3,3}
\end{array}\right] \begin{gathered}
l i \\
l(i-1)
\end{gathered}
$$

Thus the past and future outputs can be now defined by means of the submatrices of $R$ :

$$
\begin{aligned}
R_{p} & =\left[\begin{array}{lll}
R_{1,1} & 0 & 0
\end{array}\right] \quad \in R^{l i x 2 l i} \\
R_{f} & =\left[\begin{array}{llc}
R_{2,1} & R_{2,2} & 0 \\
R_{3,1} & R_{3,2} & R_{3,3}
\end{array}\right] \quad \in R^{l i x 2 l i}
\end{aligned}
$$

Step 2. The second step of the algorithm depicts the orthogonal projection of the row space of the matrix $R_{f}$ on the row space of the matrix $R_{p}$ :

$$
\begin{aligned}
O_{i} & \stackrel{\text { def }}{=} R_{f} / R_{p} \\
& =R_{f} \cdot R_{p}^{T}\left(R_{p} R_{p}^{T}\right)^{\dagger} \cdot R_{p}
\end{aligned}
$$

Step 3. Calculation of SVD of the weighted orthogonal projection:

$$
W_{1} O_{i} W_{2}=U S V^{T}
$$

The user-defined weighting matrices $W_{1}$ and $W_{2}$ are based on the canonical variate algorithm (CVA) described in [28-30] with:

$$
\begin{aligned}
& W_{1}=\Phi_{\left[R_{f}, R_{f}\right]}^{-1 / 2}=\left(R_{f} R_{f}^{T}\right)^{-1 / 2} \in R^{l i x l i}, \\
& W_{2}=I_{2 l i} \in R^{2 l i x 2 l i}
\end{aligned}
$$

where $\Phi_{\left[R_{f}, R_{f}\right]}$ is covariance of the future output matrix $R_{f}$. The CVA of calculates the principal angles and directions between the row spaces of the past outputs $R_{p}$ and the future outputs $R_{f}$. Thus, the singular value decomposition of the weighted orthogonal projection becomes:

$$
\begin{aligned}
W_{1} O_{i} & =\Phi_{\left[R_{f}, R_{f}\right]}^{-1 / 2} O_{i}, \\
& =U S V^{T} .
\end{aligned}
$$

Step 3. Determine the model order $n$ by inspecting the singular values

The singular values can be divided into two parts: Dominating part $S_{1}$ and insignificant part $S_{2}$ :

$$
W_{1} O_{i}=\left[\begin{array}{ll}
U_{1} & U_{2}
\end{array}\right]\left[\begin{array}{cc}
S_{1} & 0 \\
0 & S_{2}
\end{array}\right]\left[\begin{array}{l}
V_{1}^{T} \\
V_{2}^{T}
\end{array}\right],
$$

where

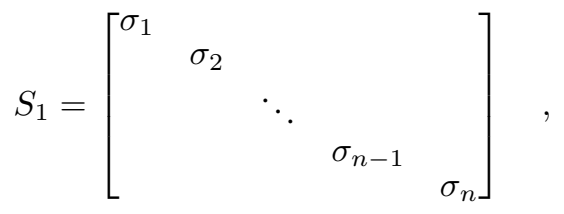

$$
S_{2}=\left[\begin{array}{ccccc}
\sigma_{n+1} & & & & \\
& \sigma_{n+2} & & & \\
& & \ddots & & \\
& & & \sigma_{l i-1} & \\
& & & & \sigma_{l i}
\end{array}\right]
$$

with $\sigma_{1} \geq \sigma_{2} \geq \cdots \geq \sigma_{n}>0$ and $\sigma_{n} \gg \sigma_{n+1} \geq$ $\sigma_{n+2} \geq \cdots \geq \sigma_{l i}$.

Step 4. Define the extended observability matrix $\Gamma_{i}$ :

$$
\Gamma_{i}=W_{1}^{-1} U_{1} S_{1}^{1 / 2}
$$

By removing the last $l$ (number of outputs) rows of $\Gamma_{i}$, we get the shifted observability matrix $\Gamma_{i-1}$ :

$$
\Gamma_{i-1}=\underline{\Gamma}_{i}
$$

Step 5. For the computation of the system matrices $A$ and $C$ the solution of the following set of linear equations is needed:

$$
T_{l}=\left[\begin{array}{l}
A \\
C
\end{array}\right] T_{r}
$$

where left-hand side of the Equation (63) is equal to:

$$
T_{l}=\left[\begin{array}{c}
\Gamma_{i}^{\dagger} R_{[3: 3],[1: 2]} \\
R_{[2: 2],[1: 2]}
\end{array}\right]=\left[\begin{array}{cc}
\Gamma_{i}^{\dagger}\left[R_{3,1}\right. & \left.R_{3,2}\right] \\
{\left[R_{2,1}\right.} & R_{2,2}
\end{array}\right]
$$

The submatrices $R_{2,1}, R_{2,2}, R_{3,1}, R_{3,3}$ are given in the Equation (52). The right-hand side of the Equation (63) is defined as follows:

$$
T_{r}=\Gamma_{i}^{\dagger} R_{[2: 3],[1: 2]}=\Gamma_{i}^{\dagger}\left[\begin{array}{ll}
R_{2,1} & R_{2,2} \\
R_{3,1} & R_{3,2}
\end{array}\right]
$$

Now, the Equation (63) can be solved by means of leastsquares method:

$$
\left[\begin{array}{l}
A \\
C
\end{array}\right]=T_{l} T_{r}^{\dagger}
$$




\section{Determination of the Modal Parameters}

Once the matrices $A$ and $C$ are estimated, the modal parameters could be easily calculated by means of an eigendecomposition of the dynamic matrix $A$ :

$$
A=\Phi \Lambda \Phi^{-1}
$$

where the diagonal matrix $\Lambda$ contains the discrete-time eigenvalues $\mu_{r}$ with $r=1,2, \ldots, n$ and the matrix $\Phi \in \mathbb{C}^{\ltimes} \curvearrowleft \ltimes$ consists of complex eigenvectors $\phi_{r}$ which appear in complex conjugate pairs:

$$
A \phi_{r}=\mu_{r} \phi_{r}
$$

The discrete eigenvalues $\mu_{r}$ can be transformed into continuous eigenvalues or system poles $\lambda_{r}$ by using the following definition:

$$
\mu_{r}=e^{\lambda_{r} \Delta t} \quad \Rightarrow \quad \lambda_{r}=\frac{1}{\Delta t} \ln \left(\mu_{r}\right) .
$$

The modal parameters $f_{r}$ (undamped eigenfrequencies) and $\zeta_{r}$ (damping ratios) can now be determined as follows:

$\lambda_{r}=-\zeta_{r} \omega_{r}+i \omega_{r} \sqrt{1-\zeta_{r}^{2}} \Rightarrow f_{r}=\frac{\left|\lambda_{r}\right|}{2 \pi}, \zeta_{r}=-\frac{\mathfrak{R e}\left(\lambda_{\mathfrak{r}}\right)}{\left|\lambda_{r}\right|}$.

The experimental (complex) mode shape $\psi_{r}$ of the $r^{\text {th }}$ mode at the sensor locations are the observed (measured) parts of the system eigenvectors $\phi_{r}$. Thus, the extracted experimental mode shape $\psi_{r}$ can be determined as follows:

$$
\psi_{r}=C \phi_{r} \quad, \quad r=1,2, \ldots, n .
$$

Stabilization Diagram for rSSI method-In case of experimental or operational system identification methods, one typically uses a model order which is significantly larger than present in the data to ensure that all dynamics of the structure are captured (overestimation of the system). The reason for this is that, due to noise and modeling inaccuracies, it often happens that no clear gap exists in the sequence of the singular values of the weighted orthogonal projection matrix given in Equation (57), which results in serious difficulties for the determination of the correct model order. In any case overestimating of the system introduces spurious poles, which have to be separated from the physical ones. Therefore, an essential part in modal parameter estimation methods comes into effect - a so-called stabilization diagram. Here, the poles associated to a given model order are compared with those obtained from a one-order lower model. Only the poles that fulfill all user-defined stabilization criteria are labeled as stable. Typical stability criteria are defined by the following inequalities [31]:

$$
\begin{gathered}
\frac{\left|f_{n+1}^{(k)}-f_{n}^{(i)}\right|}{f_{n}^{(i)}} \leqq 0,01, \\
\frac{\left|\zeta_{n+1}^{(k)}-\zeta_{n}^{(i)}\right|}{\zeta_{n}^{(i)}} \leqq 0,05, \\
1-M A C\left(\phi_{n+1}^{(k)}, \phi_{n}^{(i)}\right) \leqq 0,02,
\end{gathered}
$$

with $k=1,2, \ldots, n+1, i=1,2, \ldots, n$. In other words, Equation (72) implies that the estimated natural frequency $f^{k}$ from the system of order $(n+1)$ is frequency stable with respect to the natural frequency $f^{i}$ obtained from the system of order $(n)$ if the Equation (72) is fullfilled. Similar criteria on damping ratios and mode shapes are defined in Equation (73) and (74), respectively. If all the criteria expressed by the previous inequalities are satisfied, the pole is labeled as stable.

In the case of automated system identification methods, clustering techniques enables the automatic selection of physical poles from the stabilization diagram. Some of the most widely used algoritms are, inter alia, the Hierarchical Clustering (HC) algorithm [32], the Genetic Algorithm GA [33] and Fuzzy C-Means clustering (FCM) [34]. In the following section a suitable Hierarchical Clustering algoritm used in this study is presented.

Hierarchical Clustering for Automated rSSI-In this study the hierarchical clustering algorithms is applied on the data obtained from the stabilization diagram. Hierarchical clustering classifies data over a variety of scales by generation a cluster tree or dendrogram. The tree is a kind of a multilevel hierarchy, where clusters at one level are joined as clusters at the next level, where each member (object) of the data set itself depicts a cluster. The algorithm starts with the two clusters which are closest according to a user-defined metric distance (i.e. Euclidean distance), are merged into a single cluster (agglomerative clustering). This procedure is carried out until all of the objects are agglomerated into one hierarchically constructed cluster as illustrated in Figure 17 [35]. Different approaches of the hierarchical clustering algorithm can be used for the identification of physical poles. The substantial difference between between these approaches lies in the definition of the criteria used to quantify the degree of similarities of the mean properties of two clusters. Several methods can be found in the relevant literature to measure these similarities, e.g. [36] and [37]. Within the framework of this study following criteria are defined to quantify the similarities between two cluster centres:

$$
F(i, j)=\frac{\left|f_{i}-f_{j}\right|}{f_{j}}+1-M A C\left(\phi_{i}, \phi_{j}\right)
$$

where $F(i, j)$ is a scalar quantitiy that measure the degree of similarity between the eigenfrequencies and mode shapes of two distinct poles (or objects) denoted by indexes $i$ and $j$. Due to the agglomerated type of the Hierarchical Clustering method where clusters at one level are joined as clusters at the next level, indices $i$ and $j$ can also denote clusters consisting of more than one eigenfrequency and corresponding mode shape - in which case $f_{i}$ and $f_{j}$ are the mean eigenfrequencies of the clusters $i$ and $j$ and $\phi_{i}$ and $\phi_{j}$ are, respectively, the mean mode shapes of the clusters $i$ and $j$. A value close to zero obtained from $F(i, j)$ indicates a high similarity of the mean properties of two clusters of poles denoted by indices $i$ and $j$. The Modal Assurance Criterion (MAC) from the Equation (76) is a measure of the degree of linearity between two vectors. Given two vectors $\phi_{i}, \phi_{i}$, it is defined by following formula:

$$
\operatorname{MAC}\left(\phi_{i}, \phi_{j}\right)=\left(\frac{\left|\phi_{i}^{H} \phi_{j}\right|}{\left\|\phi_{i}\right\|\left\|\phi_{j}\right\|}\right)^{2}
$$

where ()$^{H}$ designate the Hermetian transpose of a complex vector. This criterion can be applied to both real-valued and complex-valued vectors. In this context, it indicates the correlation degree between the two mode shapes $\phi_{i}$ and $\phi_{j}$. 


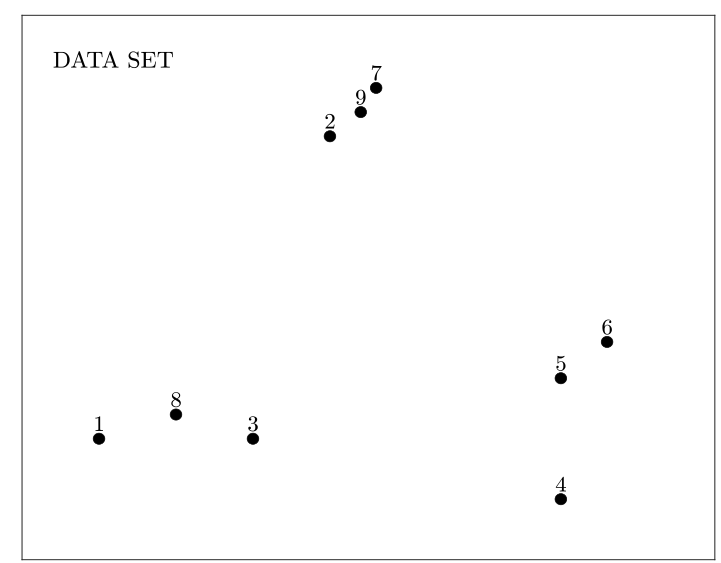

(a)

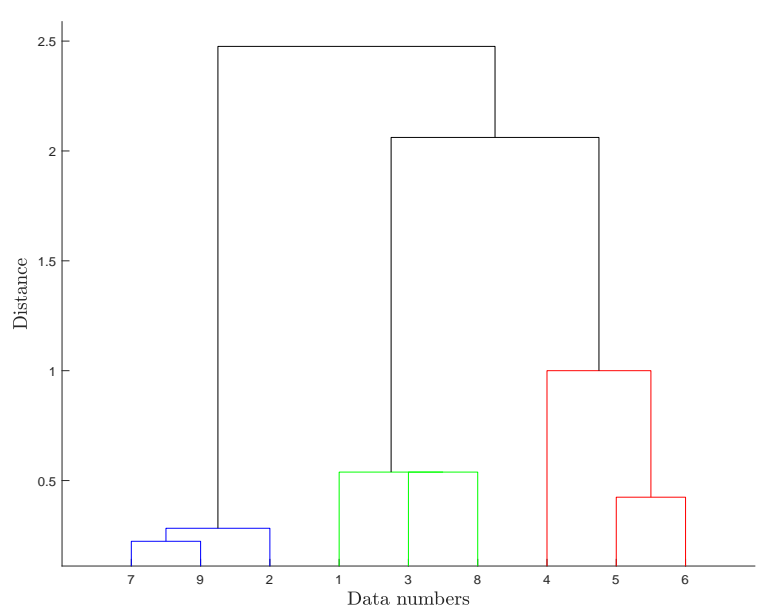

(b)

Figure 17: Illustration of agglomerative clustering: Data set (a) and corresponding hierarchical clustering dendrogram (b)

\section{Application of rSSI method to in-flight aircraft data}

In this section the application of the rSSI method to in-flight data of the FliPASED aircraft will be presented. The introduced rSSI algorithm is coded within MATLAB environment. For the identification of the flight modal parameters of the FLIPASED aircraft, the wings were instrumented with twelve inertial measurement units (IMU) with the help of which the structural vibrations of the wings are detected. At 30\%, 60\% and $90 \%$ span width of each wing an IMU is attached to the front and to the rear spar as shown in Figure 9. The IMUs measure the translational accelerations in $z$ direction and the angular rates $\omega_{x}$ and $\omega_{y}$. Therefore, 36 raw time histories ( $12 \times 3$ channels) are available for the data analysis. The measured vibrations were the structural response to the natural operational excitation, being mainly the atmospheric turbulence and, additionally, to the pulse excitation by means of doublet input via ruddervators. The flight test where the recorded time series have been taken from was performed at an altitude of $663 \mathrm{~m}$ (MSL) -and with a flight speed of $34 \mathrm{~m} / \mathrm{s}$.
Preprocessing of the data set and input parameters for rSSI method-The in-flight raw test data consisting of translational acceleration and angular velocitiy time series are analysed within the time window of 11,54 seconds. A bandpass Butterworth filter of 4th-order is applied to all measured responses with a lower cutoff frequency of $0,5 \mathrm{~Hz}$ and a higher cutoff frequency of $45 \mathrm{~Hz}$. The original time histories were sampled at $200 \mathrm{~Hz}$ and are then downsampled to 100 $\mathrm{Hz}$ where computational effort can be reduced to a minimum. The whole preprocess required only 1.507 seconds of computation time.

For the rSSI analysis following parameters are chosen:

- Number of block rows in Hankel matrices: $N_{i}=12$

- Max. frequency deviation: $\Delta_{\text {freq }}=1,25 \%$

- Max. damping ratio deviation: $\Delta_{\text {damp }}=5 \%$

- Min. MAC value: $M A C_{\min }=95 \%$

- Model order range: $n=\left[\begin{array}{ll}\min & 65\end{array}\right]$

- Threshold for inconsistency coefficient within cluster analyis: $C_{\text {incons }}=0,4$

After definition of the input parameters outlined above, the rSSI method has been applied to the in-flight aircraft data. The obtained results are presented in detail in the next section.

Identification results obtained with rSSI-The rSSI algorithm is applied to the prepocessed in-flight data for the modal identification. The identified flight modal parameter from test are then compared with the predicted results provided by the numerical model by means of modal correlation analysis. Table 5 summerizes both the obtained modal parameters and the correlation results. The four identified modes consisting of $2^{\text {nd }}, 3^{\text {rd }}, 4^{\text {th }}$ and $6^{\text {th }}$ wing bending within the frequency range of $3 \mathrm{~Hz}<\mathrm{f}<30 \mathrm{~Hz}$ could be identified from the flight test data. In this context, it is important to mention here that the $5^{\text {th }}$ wing bending mode could not be extracted from the available test data due to poor excitation as can be seen from the stabilization diagram as illustrated in Figure 18.

The flight modal parameters computed from the flexible aircraft model show an excellent correlation compared with those identified from flight test with MAC values $>95 \%$, frequency deviations $<3,5 \%$ and almost perfect matching between damping ratios with a maximum deviation of $<0,7$ $\%$, as can be seen in the Table 5 . The introduced rSSI method

Table 5: Comparison of flight modal parameters obtained from flight test and numerical model

\begin{tabular}{|c|c|c|c|c|}
\hline Mode & 1 & 2 & 3 & 4 \\
\hline$f_{\text {Test }}[\mathrm{Hz}]$ & 3.3 & 8.5 & 12.3 & 26.7 \\
\hline$f_{\text {Model }}[\mathrm{Hz}]$ & 3.4 & 8.3 & 11.9 & 27.1 \\
\hline$\Delta_{\text {freq }}[\%]$ & +3.5 & -2.1 & -2.6 & +1.6 \\
\hline$\zeta_{\text {Test }}[\%]$ & 14.2 & 6.4 & 4.3 & 3.8 \\
\hline$\zeta_{\text {Model }}[\%]$ & 14.1 & 6.4 & 4.3 & 3.8 \\
\hline$\Delta_{\text {damp }}[\%]$ & -0.7 & +0.0 & +0.0 & +0.0 \\
\hline MAC [\%] & 99 & 98 & 99 & 95 \\
\hline
\end{tabular}

for estimation of flight modal parameters shows a high degree of robustness even in the presence of limited amounts of data and high noise in the measurement. The cleaned stabilization diagram in Figure 19 (b) obtained by the rSSI method where the extracted physical modes are shown after hierarchical clustering, clearly illustrate this. Finally, the identified mode shapes from the flight test and their counterparts from the numerical model are visualized in Figures 20 to 27. 


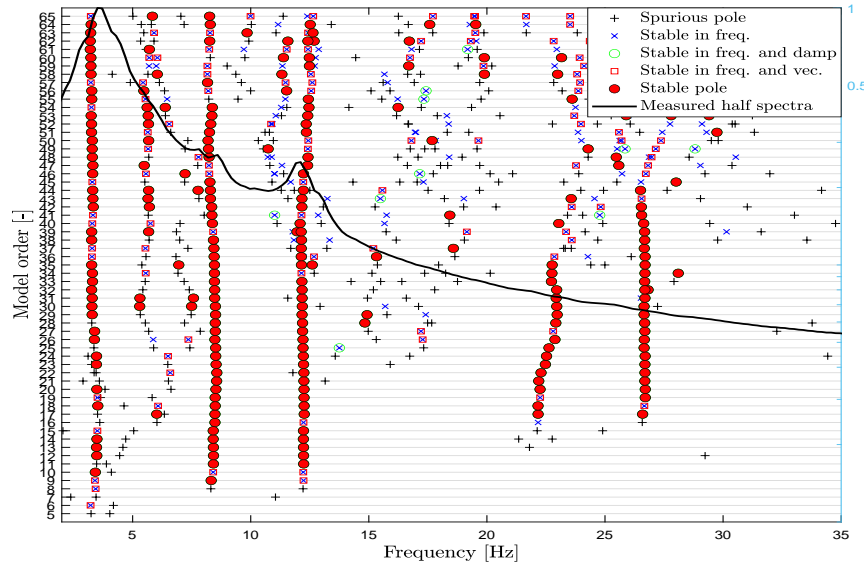

Figure 18: Uncleaned rSSI-stabilization diagram obtained from flight test

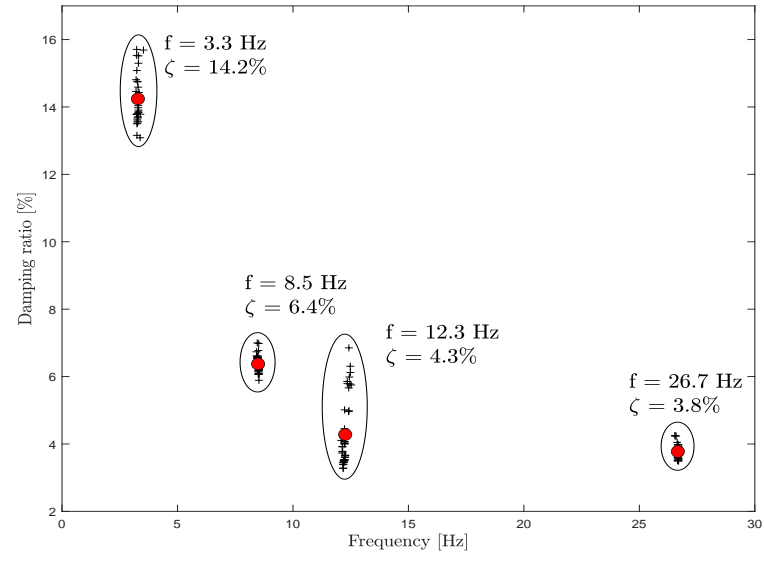

(a)

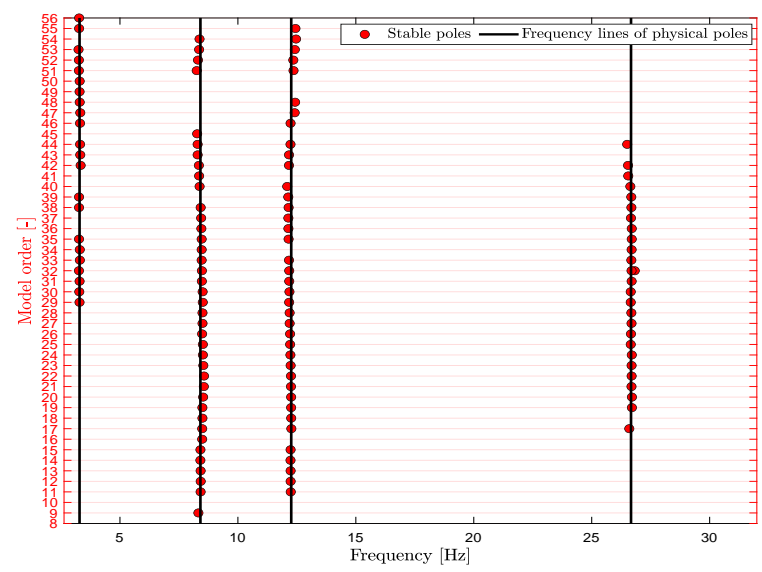

(b)

Figure 19: Cluster plot of identified physical poles (a) Cleaned rSSI stabilization diagram from obtained flight test after cluster analysis (b)

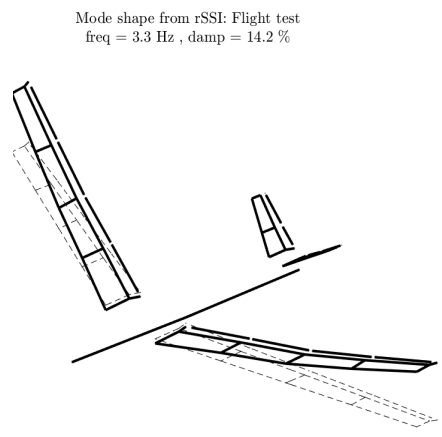

Mode shape from flexible aircraft model
freq $=3.4 \mathrm{~Hz}$, damp $=14.1 \%$

Figure 20: $2^{\text {nd }}$ wing bending (flight test)

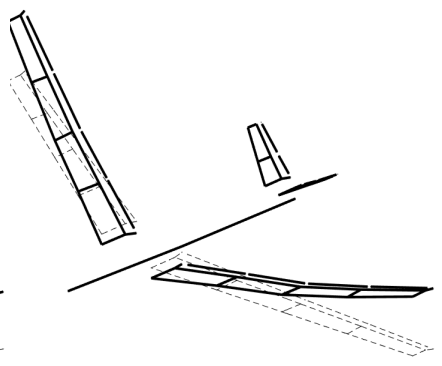

Figure 21: $2^{\text {nd }}$ wing bending (aircraft model)

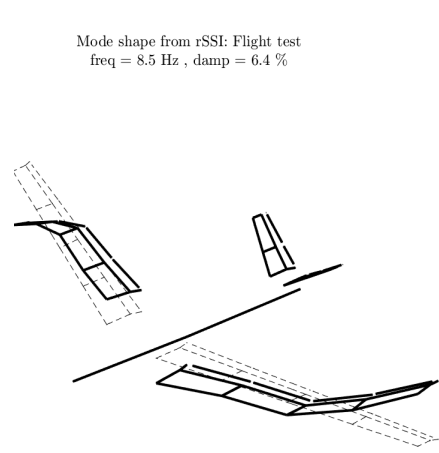

Figure 22: $3^{\text {rd }}$ wing bending (flight test) Mode shape from flexible aircraft model
freq $=8.3 \mathrm{~Hz}$, damp $=6.4 \%$

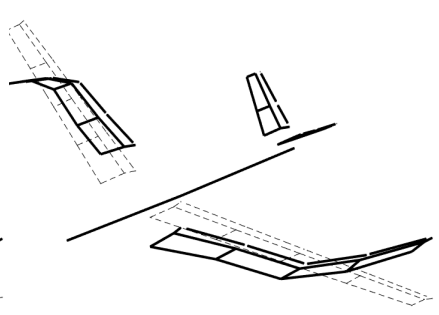

Figure 23: $3^{\text {rd }}$ wing bending (aircraft model)
Mode shape from rSSI: Flight test
freq $=12.3 \mathrm{~Hz}$, damp $=4.3 \%$

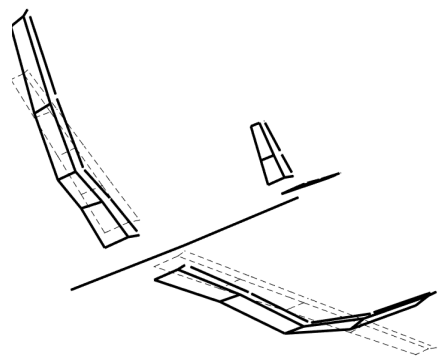

Figure 24: $4^{\text {th }}$ wing bending (flight test) Mode shape from flexible aircraft model
freq $=11.9 \mathrm{~Hz}$, damp $=4.3 \%$

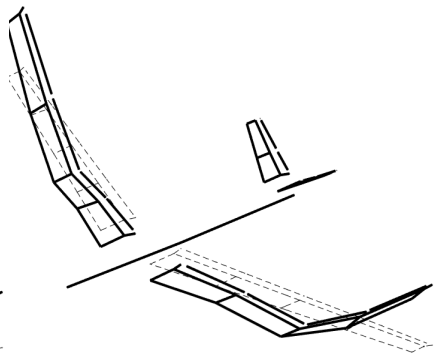

Figure 25: $4^{\text {th }}$ wing bending (aircraft model) 

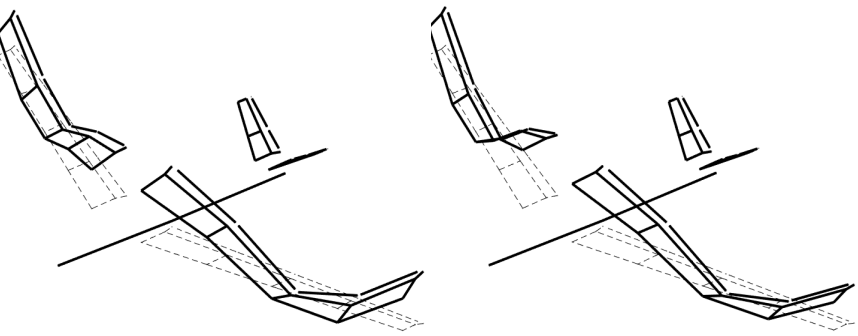

Figure 26: $6^{\text {th }}$ wing bending (flight test)

Figure 27: $6^{\text {th }}$ wing bending (aircraft model)

\section{SUMMARY}

The aeroservoelastic modelling approach has proven appropriate for the considered demonstrator aircraft. The rigid body model can be further improved with the output error method (OEM) by analysing specific flight test manoeuvres. However, to keep disturbances at a minimum flight tests should take place during a calm day.

A separated update of longitudinal and lateral parameters of the flight mechanical model is promising. A simulation of the model featuring the updated parameters shows a good agreement with the flight test data. Nevertheless, the rigid body model can still be improved. So far the parameters of the drag polar were assumed as given, although they are based on preliminary design methods. In a next step, the drag polar parameters will be updated as well. Furthermore, the model could be extended to provide forces in $x$-direction, which would lead to a further improvement of the flight mechanical model.

For the flexible motion, an automatically running robust Stochastic Subspace Identification (rSSI) method is used for the identification of the flight modal paramerters, which are often used for quality assessment of the actual numerical model and consequently its validation with respect to the flexible aircraft dynamics. The application of the rSSI method to the in-flight data has been successfully demonstrated. The identified flight modal parameters were then compared with the predicted counterparts computed from the numerical model by means of modal correlation analysis.

\section{ACKNOWLEDGMENTS}

The research leading to these results is part of the FLiPASED project. This project has received funding from the European Unions Horizon 2020 research and innovation program under grant agreement No. 815058 .

\section{REFERENCES}

[1] International Energy Agency, Transport Energy and CO2: Moving Towards Sustainability. OECD Publishing, 2009.

[2] E. H. Dowell, A modern course in aeroelasticity. Springer, 2014, vol. 217.

[3] R. Bisplinghoff, H. Ashley, and R. Halfman, "Aeroelasticity," Addison-Wes-ley Pub. Co, 1955.

[4] FLEXOP Consortium, "The flexop project," URL:

https://flexop.eu/, 2015.

[5] CORDIS, "Flight phase adaptive aero-servo-elastic aircraft design methods I flipased project I h2020 | cordis I european commission," 14.10.2020. [Online]. Available: https://cordis.europa.eu/project/id/815058

[6] M. Pusch, D. Ossmann, and T. Luspay, "Structured control design for a highly flexible flutter demonstrator," Aerospace, vol. 6, no. 3, p. 27, 2019.

[7] T. Luspay, D. Ossmann, M. Wuestenhagen, D. Teubl, T. Baár, M. Pusch, T. M. Kier, S. Waitman, A. Ianelli, A. Marcos, B. Vanek, and M. H. Lowenberg, "Flight control design for a highly flexible flutter demonstrator," in AIAA Scitech 2019 Forum. Reston, Virginia: American Institute of Aeronautics and Astronautics, 01072019, p. 1.

[8] S. Waitman and A. Marcos, " $\mathrm{H} \infty$ control design for active flutter suppression of flexible-wing unmanned aerial vehicle demonstrator," Journal of Guidance, Control, and Dynamics, vol. 43, no. 4, pp. 656-672, 2020.

[9] M. Wuestenhagen, T. Kier, Y. M. Meddaikar, M. Pusch, D. Ossmann, and A. Hermanutz, "Aeroservoelastic modeling and analysis of a highly flexible flutter demonstrator," in 2018 Atmospheric Flight Mechanics Conference. Reston, Virginia: American Institute of Aeronautics and Astronautics, 06252018.

[10] T. Kier and G. Looye, "Unifying manoeuvre and gust loads analysis," International Forum on Aeroelasticity and Structural Dynamics, 2009.

[11] R. V. Jategaonkar, Flight Vehicle System Identification. Reston: American Institute of Aeronautics and Astronautics, 2015.

[12] A. Tewari, Aeroservoelasticity - Modeling and Control. Springer-Verlag, 2015.

[13] E. Ferede and M. Abdalla, "Cross-sectional modelling of thin-walled composite beams," in 55th AIAA/ASMe/ASCE/AHS/SC Structures, Structural Dynamics, and Materials Conference, 2014.

[14] R. J. Guyan, "Reduction of stiffness and mass matrices," AIAA Journal, 1964.

[15] C. Reschke, "Integrated flight loads modelling and analysis for flexible transport aircraft," Ph.D. dissertation, University of Stuttgart, 2006.

[16] J. Hofstee, T. Kier, C. Cerulli, and G. Looye, "A variable, fully flexible dynamic response tool for special investigations (varloads)," International Forum on Aeroelasticity and Structural Dynamics, 2003.

[17] Thiemo Kier, "An integrated model for lateral gust loads analysis and dutch roll flight dynamics using a $3 \mathrm{~d}$ panel method," in International Forum on Aeroelasticity and Structural Dynamics, 2017. [Online]. Available: https://elib.dlr.de/118398/

[18] P. Stahl, F.-M. Sendner, A. Hermanutz, C. Rößler, and M. Hornung, "Mission and aircraft design of flexop unmanned flying demonstrator to test flutter suppression within visual line of sight," 17th AIAA Aviation Technology, Integration, and Operations Conference, 2017.

[19] T. Kier and J. Hofstee, "Varloads-eine simulationsumgebung zur lastenberechnung eines voll flexiblen, freifliegenden flugzeugs," in Deutscher Luft-und Raumfahrtkongress, 2004.

[20] K. L. Roger, "Airplane math modeling methods for active control design," AGARD-CP-228, 1977. 
[21] Manuel Pusch, Blending of Inputs and Outputs for Modal Control of Aeroelastic Systems: Dissertation. München: Verlag Dr. Hut, 2020.

[22] "Edmo airport." [Online]. Available: https: //edmo-airport.de/

[23] J. Sodja, R. de Breuker, Y. M. Meddaikar, J. K. Dillinger, K. Soal, Y. Govers, W. Krueger, P. Georgopoulos, C. Koimtzoglou, C. Roessler, S. J. Koeberle, J. Bartasevicius, D. Teubl, L. Gyulai, S. Toth, M. Nagy, D. Balogh, M. Jasdi, P. Bauer, and B. Vanek, "Ground testing of the flexop demonstrator aircraft," in AIAA Scitech 2020 Forum. Reston, Virginia: American Institute of Aeronautics and Astronautics, 01062020, p. 1.

[24] J. Sodja, N. Werter, and R. de Breuker, "Design of a flying demonstrator wing for manoeuvre load alleviation with cruise shape constraint," in 2018 AIAA/ASCE/AHS/ASC Structures, Structural Dynamics, and Materials Conference. Reston, Virginia: American Institute of Aeronautics and Astronautics, 01082018, p. 1 .

[25] V. Klein and E. A. Morelli, Aircraft System Identification: Theory and Practice. Reston ,VA: American Institute of Aeronautics and Astronautics, 2006.

[26] J. A. Mulder, Q. P. Chu, J. K. Sridhar, J. H. Breeman, and M. Laban, "Non-linear aircraft flight path reconstruction review and new advances," Progress in Aerospace Sciences, vol. 35, no. 7, pp. 673-726, 1999.

[27] S. Amador, "Uncertainty quantification in operational modal analysis and continuous monitoring of special structures, phd thesis," in Faculty of Engineering of University of Porto for the degree of Doctor in Civil Engineering, 2015, p. 79. [Online]. Available: https://repositorio-aberto.up.pt/bitstream/ 10216/79102/2/35163.pdf

[28] P. Van Overschee and B. De Moor, Subspace identification for linear systems: Theory-Implementation-Applications. Springer US, 1996.

[29] K. Arun and S. Kung, Balanced approximation of stochastic systems. SIAM J. Matrix Analysis and Applications, 11, 1990.

[30] P. Van Overschee and B. De Moor, Subspace algorithms for the stochastic identification problem. Automatica, Vol. 29, no. 3, 1993.

[31] C. Rainieri and G. Fabbrocino, Operational Modal Analysis of Civil Engineering Structures, An introduction and a guide for applications, 2014.

[32] F. Magalhaes, A. Cunha, and E. Caetano, "Online automatic identification of the modal parameters of a long span arch bridge," in Mechanical Systems and Signal Processing, vol. 23, 2009, pp. 316-329.

[33] C. Jung-Huai and G. Jamshid, "Genetic algorithm in structural damage detection," Computers and Structures, vol. 79, no. 14, pp. 1335-1353, 2001. [Online]. Available: http://www.sciencedirect.com/ science/article/pii/S004579490100027X

[34] E. P. Carden and J. M. W. Brownjoh, "Fuzzy clustering of stability diagrams for vibration-based structural health monitoring," Computer-Aided Civil and Infrastructure Engineering, vol. 23, no. 5, pp. 360-372, 2008.

[35] S. Amador, "Uncertainty quantification in operational modal analysis and continuous monitoring of special structures, phd thesis," in Faculty of Engineering of University of Porto for the degree of Doctor in Civil Engineering, 2015, p. 152. [Online]. Available: https://repositorio-aberto.up.pt/bitstream/ 10216/79102/2/35163.pdf

[36] F. Magalhaes, A. Cunha, and E. Caetano, "Online automatic identification of the modal parameters of a long span arch bridge," in Mechanical Systems and Signal Processing, vol. 23, no. 2, 2009, pp. 316-329.

[37] E. Reynders, J. Houbrechts, and G. De Roeck, "Fully automated (operational) modal analysis," in Mechanical Systems and Signal Processing, vol. 29, 2012, pp. 228250.

\section{BIOGRAPHY}

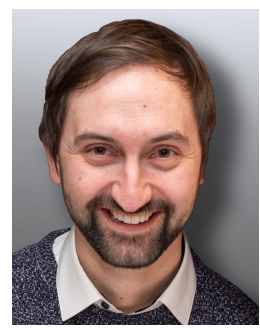

Matthias Wüstenhagen received his B.S. and M.S. degrees in Aerospace Engineering from the University of Stuttgart in 2016. Ever since he is a research associate at the Institute of System Dynamics and Control of the German Aerospace Center (DLR). His current research activities include modelling and control of flexible aircraft.

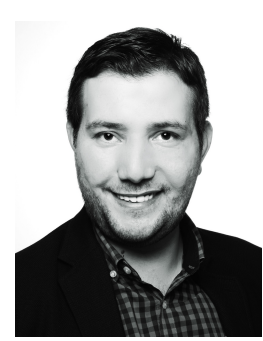

Özge Süelözgen received his Diplom (equivalent to M.Sc.) degree in Aerospace Engineering from the Technical University of Berlin in 2013. Currently he is a research associate at the Institute of System Dynamics and Control of the German Aerospace Center (DLR). His current research activities are focussed on modelling and developing of methods for system identification of flexible aircraft structures.

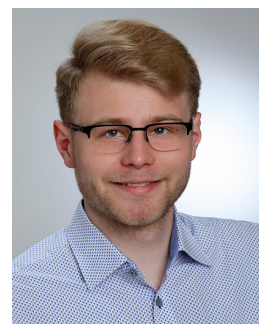

Lukas Ackermann received his B.S. degree in Aerospace Engineering from the University of Stuttgart. Presently he is working at the Institute of System Dynamics and Control of DLR to finish his M.S. studies in Aerospace Engineering at the University of Stuttgart. His work is focused on flexible aircraft model parameter estimation.

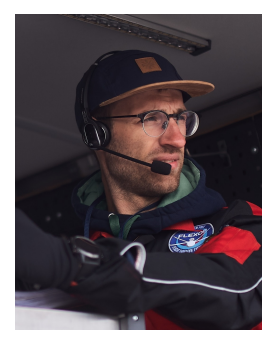

Julius Bartaševičius has graduated from the University of Glasgow, where he received an MEng degree in Aeronautical Engineering with main focus in aerodynamics. Currently he is a research assistant within the Technical University of Munich, Institute of Aircraft design. Flight testing and instrumentation of UAVs are his main research areas. 